\title{
Subspace Methods for Self-Calibration of ULAs with Unknown Mutual Coupling: A False-Peak Analysis
}

\author{
Shu Cai ${ }^{\mathrm{a}}$, Jun Zhang ${ }^{\mathrm{a}, *}$, Gang Wang ${ }^{\mathrm{b}}$, Hongbo Zhu ${ }^{\mathrm{a}}$, Kai-Kit Wong ${ }^{\mathrm{c}}$ \\ ${ }^{a}$ Jiangsu Key Lab of Wireless Communication, Nanjing University of Posts and \\ Telecommunications, Nanjing, 230001 China \\ ${ }^{b}$ Faculty of Electrical Engineering and Computer Science, Ningbo University, Ningbo, China \\ ${ }^{c}$ Department of Electronic and Electrical Engineering, University College London, London, \\ WC1E 7JE, United Kingdom
}

\begin{abstract}
Self-calibration can mitigate the adverse effect of array imperfections in directionof-arrival (DoA) estimation. In this paper, we consider self-calibration of uniform linear arrays (ULAs) with unknown mutual coupling and focus on subspacebased methods. By modeling the effect of mutual coupling through complex symmetric Toeplitz matrices, we first analyze its influence to existing subspace methods. Our analytical results illustrate that mutual coupling can induce false peaks in spatial spectra of these methods, and more importantly, most of these peaks are predictable. Based on the analytical results, we then propose new spectra to suppress these predictable false peaks, and new self-calibration algorithms for the proposed spectra. Simulations are performed to validate our analytical results and to illustrate the advantages of the proposed algorithms.
\end{abstract}

Keywords: Direction-of-arrival (DoA) estimation, uniform linear array

(ULA), mutual coupling, array self-calibration, subspace method

\section{Introduction}

Direction finding (DF) with antenna arrays is widely adopted in areas of radar, sonar, and wireless communications. In particular, high resolution DF

\footnotetext{
* Corresponding author

Email address: zhangjun@njupt.edu.cn (Jun Zhang)
}

Preprint submitted to Journal of ${ }^{A} T_{E} X$ Templates

October 8, 2019 
techniques [1] can achieve excellent direction-of-arrival (DoA) estimation perfor-

5 mance when the array response is perfectly known. In a practical array, electromagnetic characteristics cause sensor responses that always interfere with each other, resulting in mutual couplings between sensors [2]. Results in the literature [3, 4] have already illustrated that ignoring the effect of mutual coupling can seriously degrade the estimation accuracy of high-resolution algorithms.

To eliminate the effect of mutual coupling, one approach is to design the array structure carefully. For example, sparse arrays [5] enjoy weaker mutual coupling than uniform linear arrays (ULAs). However, a good structure can only relieve but not remove mutual coupling.

Another technique involves modeling and parameterizing the effect of unknown mutual coupling, estimating the obtained parameters, and then compensating them in DF algorithms. This can be achieved by using reference sources at known positions [6, 7]. However, these methods are usually computationally expensive, time consuming [8], and not adaptive to ongoing changes in the environment 9. Therefore, jointly estimating the effect of mutual coupling and DoA, which is referred to as self-calibration, has attracted much attention.

The goal of this paper is to design self-calibration schemes for ULAs with unknown mutual coupling effect. In ULAs, this effect can be modeled as a symmetric Toeplitz mutual coupling matrix (MCM) [4, 10, 20]. In [10 12], the authors illustrated that by resorting to a middle subarray (mid-array) of a ULA, 25 accurate DoA estimation can be obtained without mutual coupling compensation. However, its application is limited due to the need of a large number of auxiliary sensors. Iterative algorithms were also proposed [13 15] but the performance of non-convex iterative approaches depends on their initializations. Another method is the use of a rank reduction (RARE) estimator [16 18, 21]. But the unknowns in MCM may induce false peaks in their spatial spectra. To remove the influence of false peaks, a recursive-RARE (R-RARE) [19] method and a blind method [20], which are based on multidimensional spectra, were proposed. Unfortunately, both methods may still suffer from false peaks [22]. In general, iterative methods and RARE methods are heuristic in the sense that 
they do not rigorously analyze the impact of unknown MCM to the estimation performance, and thus are sensitive to initial values or false peaks.

In this paper, we aim to remove the ambiguities by false peak analysis. We first analyze the influence of the MCM to spatial spectra of some existing subspace-based methods, such as those in [4, 17+20]. Then, based on the 40 analytical results, we propose new spatial spectra as well as the corresponding self-calibration algorithms. Our main contributions, both theoretical and technical, are summarized as follows:

1. We analyze a widely employed one-dimensional multiple signal classification (MUSIC) type spatial spectrum. The analytical result indicates that the MCM can cause serious false peaks, which are divided into two categories: predictable and unpredictable. Predictable ones are those independent of true DoAs and MCM coefficients and only induced by special structures of MCM, while unpredictable peaks are caused by special values of true DoAs or MCM coefficients. So the former may be predicted by analyzing the properties of M-

50 CM. According to the rule whether their value is affected by noise, predictable peaks can be further classified into noise independent and dependent ones. The sufficient and necessary conditions for noise independent peaks are given out. To suppress these peaks, almost all existing methods restrict the number of unknowns in MCM, by assuming that the mutual coupling between sensors spaced ${ }_{55}$ by a certain distance is negligible. However, this distance is often chosen heuristically and small, and truncating the effects of mutual coupling will inevitably give rise to modeling errors and estimation errors. Fortunately, our conditions provide a theoretical bound for choosing the distance in these methods.

2. The multidimensional spectra based calibration methods are considered.

60 We first introduce a dimension-scalable spectrum to reduce computational complexity of a widely used multidimensional spatial spectrum. Then we analyze sufficient and necessary conditions for noise independent false peaks and provide some sufficient conditions for noise dependent peaks and unpredictable peaks.

3. Moreover, we propose a one-dimensional spectrum and a multidimensional ${ }_{65}$ spectrum by utilizing the conditions for false peaks. Both spectra are improve- 
ments of existing ones and can automatically suppress predictable false peaks without affecting peaks of true DoAs. Two complexity scalable self-calibration algorithms are proposed, respectively, for the two spectra. A key difference between our work and existing methods is that ours is based on rigorous false peak analysis, which leads to advantages such as higher estimation reliability, wider application range, low and scalable computational complexity, and that truncation of the effect of mutual coupling is not essential in many situations.

In the rest of paper, section 2 introduces the signal model and reviews existing methods. Sections 3 and 4 analyze the influence of MCM to one-dimensional and multidimensional spectra, respectively. Section 5 proposes new algorithms. Sections 6 and 7 present numerical results and conclusion, respectively.

Notations - For a given matrix $\boldsymbol{X}$, we use $\boldsymbol{X}^{T}, \boldsymbol{X}^{H}, \operatorname{rank}(\boldsymbol{X})$, and $\lambda_{i}(\boldsymbol{X})$ to denote its transpose, conjugate transpose, rank, and the $i$-th eigenvalue in ascending order, respectively. In addition, $\|\boldsymbol{x}\|$ and $\|\boldsymbol{X}\|$ denote the $l_{2}$-norm and Frobenius norm of $\boldsymbol{x}$ and $\boldsymbol{X}$, respectively. The diagonalization and block diagonalization operators are $\operatorname{diag}(\cdot)$ and $\operatorname{blkdg}\{\cdot\}$, respectively. Also, $\lceil x\rceil$ (resp. $\lfloor x\rfloor$ ) rounds $x$ to the nearest integer greater (resp. smaller) than itself. For an integer $N,[N] \triangleq\{1,2, \ldots, N\}$. The notations $\boldsymbol{X}_{[i, j]}$ and $\boldsymbol{X}_{[N]}$ denote the $(i, j)$ th element of $\boldsymbol{X}$ and the first $\mathrm{N}$ columns of $\boldsymbol{X}$, respectively. Also, $\boldsymbol{x}_{[N]}$ denotes first $N$ entries of $\boldsymbol{x}$. Moreover, $\boldsymbol{I}_{N}$ is an $N$-dimensional identity matrix and $\boldsymbol{J}_{N}$ is a row-reversed version of $\boldsymbol{I}_{N}$. Also, $\boldsymbol{I}_{N}^{(k)}$ is a matrix with ones on the $k$-th diagonal below the main diagonal and zeros elsewhere. $\mathbb{Z}$ and $\mathbb{C}$ stand for the sets of integers and complex numbers, respectively. Finally, Toeplitz(c) is a symmetric Toeplitz matrix with the first column $\boldsymbol{c}$.

\section{Preliminaries}

\subsection{Signal Model and Assumptions}

Consider $M$ narrowband far-field sources impinging on an $N$-element ULA from directions $\tilde{\theta}_{m}$, for $m \in[M]$. The received signals can be modeled as

$$
\boldsymbol{x}(k)=\boldsymbol{C} \boldsymbol{A} \boldsymbol{s}(k)+\boldsymbol{n}(k), \quad k=1, \ldots, N_{T},
$$


where $\boldsymbol{x}(k) \in \mathbb{C}^{N}, \boldsymbol{s}(k) \in \mathbb{C}^{M}$, and $\boldsymbol{n}(k) \in \mathbb{C}^{N}$ denote the vectors of observation$\mathrm{s}$, source signals, and observation noise, respectively, $\boldsymbol{A}=\left[\boldsymbol{a}\left(\tilde{\theta}_{1}\right), \cdots, \boldsymbol{a}\left(\tilde{\theta}_{M}\right)\right]$, $\boldsymbol{a}\left(\tilde{\theta}_{m}\right)=\left[1, e^{j \pi \beta \sin \left(\tilde{\theta}_{m}\right)}, \cdots, e^{j(N-1) \pi \beta \sin \left(\tilde{\theta}_{m}\right)}\right]^{T}$ is the steering vector of the $m$-th source. For the uniqueness of estimation, the domain of DoA is $\Omega_{\theta}=\{\theta \mid \theta \in$ $\left.\left[-\frac{\pi}{2}, \frac{\pi}{2}\right),|\beta \sin (\theta)|<1\right\}$. The MCM $\boldsymbol{C}=$ Toeplitz $(\boldsymbol{c})$ with $\boldsymbol{c}=\left[c_{1}, c_{2}, \ldots, c_{N}\right]^{T}$ [4, 13. Since the mutual coupling between two antennas is inversely proportional to their distance, we assume that $c_{1}=1 \geq\left|c_{l}\right|, \forall l \in[N]$ and there exists an $L(\leq N)$ such that for arbitrary $l>L, c_{l}=0$. Under these assumptions, a transformation holds between $\boldsymbol{C}$ and $\boldsymbol{c}[13$ :

$$
\boldsymbol{C a}(\theta)=\boldsymbol{Q}(\theta) \boldsymbol{c}=\boldsymbol{Q}_{[L]}(\theta) \boldsymbol{c}_{[L]}
$$

where $\boldsymbol{Q}(\theta)=\boldsymbol{Q}_{1}(\theta)+\boldsymbol{Q}_{2}(\theta), \boldsymbol{Q}_{1[p, q]}(\theta)=\boldsymbol{a}(\theta)_{p+q-1}$ if $p+q-1 \leq N$, $\boldsymbol{Q}_{2[p, q]}(\theta)=\boldsymbol{a}(\theta)_{p-q+1}$ if $p \geq q \geq 2$, otherwise, the entries equal 0 .

Some standard assumptions pertaining to subspace methods are used.

${ }_{95}$ (A1) $M<N$ and the columns of $\boldsymbol{C} \boldsymbol{A}$ are linearly independent.

(A2) The signals are not perfectly correlated and are uncorrelated with noise.

(A3) The noise is Gaussian distributed with zero mean and covariance $\sigma_{n}^{2} \boldsymbol{I}_{N}$.

\subsection{Existing Subspace-based Self-Calibration Methods}

Denote $\boldsymbol{R}_{x}=\mathbb{E}\left[\boldsymbol{x}(k) \boldsymbol{x}^{H}(k)\right]$. Then the matrix of singular vectors of $\boldsymbol{R}_{x}$ can be partitioned as $\left[\boldsymbol{U}_{s}, \boldsymbol{U}_{n}\right]$, where the $N \times M$ matrix $\boldsymbol{U}_{s}$ is referred to as "signal subspace" and $\boldsymbol{U}_{n}$ is "noise subspace" [4. Using the principle of subspace methods [13, we have

$$
\boldsymbol{U}_{n}^{H} \boldsymbol{C a}\left(\tilde{\theta}_{m}\right)=\boldsymbol{U}_{n}^{H} \boldsymbol{Q}_{[L]}\left(\tilde{\theta}_{m}\right) \boldsymbol{c}_{[L]}=0, \quad m \in[M] .
$$

Based on (3), an extension of the MUSIC spectrum is given by [4]

$$
P_{1}(\boldsymbol{v}, \boldsymbol{\theta})=\sum_{m=1}^{M}\left\|\boldsymbol{V} \boldsymbol{a}\left(\theta_{m}\right)\right\|^{2} /\left\|\boldsymbol{U}_{n}^{H} \boldsymbol{V} \boldsymbol{a}\left(\theta_{m}\right)\right\|^{2}
$$

where $\boldsymbol{\theta}=\left[\theta_{1}, \ldots, \theta_{M}\right]^{T}, \boldsymbol{V}=\operatorname{Toeplitz}(\boldsymbol{v})$, and $\boldsymbol{v}=\left[v_{1}, \cdots, v_{N}\right]^{T}$. Then $P_{1}(\boldsymbol{v}, \boldsymbol{\theta})$ attains its maximum when $\boldsymbol{v}^{1}$ is a scaled version of $\boldsymbol{c}$ and $\boldsymbol{\theta}=\tilde{\boldsymbol{\theta}}$, where

\footnotetext{
${ }^{1}$ To guarantee unique solution, the constraint $\|\boldsymbol{v}\|=1$ or $v_{1}=1$ can be used [4] 17, 19].
} 
$\tilde{\boldsymbol{\theta}}$ denotes the vector of true DoAs. However, searching this maximum can be computationally prohibitive even if $M$ is small. Hence, another spectrum is proposed for self-calibration in 4, 19, 20,

$$
P_{2}(\boldsymbol{\theta})=\max _{\boldsymbol{v}_{[L]},\left\|\boldsymbol{v}_{[L]}\right\|=1} \frac{1}{\sum_{m=1}^{M}\left\|\boldsymbol{U}_{n}^{H} \boldsymbol{Q}_{[L]}\left(\theta_{m}\right) \boldsymbol{v}_{[L]}\right\|^{2}}=1 / \lambda_{1}\left(\boldsymbol{W}^{H}(\boldsymbol{\theta}) \boldsymbol{W}(\boldsymbol{\theta})\right),
$$

where $\boldsymbol{W}(\boldsymbol{\theta})=\left[\boldsymbol{Q}_{[L]}^{H}\left(\theta_{1}\right) \boldsymbol{U}_{n}, \ldots, \boldsymbol{Q}_{[L]}^{H}\left(\theta_{M}\right) \boldsymbol{U}_{n}\right]^{H}$ is an $N M \times L$ matrix. According to $(3), P_{2}(\boldsymbol{\theta})$ attains its maximum if $\boldsymbol{\theta}=\tilde{\boldsymbol{\theta}}$. The advantage of $P_{2}(\boldsymbol{\theta})$ is that $\lambda_{1}$ can be easily calculated given $\boldsymbol{\theta}$. But searching the maximum of $P_{2}(\boldsymbol{\theta})$ is still costly. Hence, a one-dimensional spectrum [10, 13, 16, 19] is proposed

$$
P_{3}(\theta)=1 / \lambda_{1}\left(\boldsymbol{W}^{H}(\theta) \boldsymbol{W}(\theta)\right),
$$

where $\boldsymbol{W}(\theta)=\boldsymbol{U}_{n}^{H} \boldsymbol{Q}_{[L]}(\theta)$.

100

\section{False Peak Analysis in One-Dimensional Spectrum}

Consider the spectrum $P_{3}(\theta)$, which goes to infinity when $\theta=\tilde{\theta}_{m}, \forall m \in[M]$, according to (3). Hence, the DoA estimates based on $P_{3}(\theta)$ is statistically consistent if only the spectrum is not polluted by false peaks. Unfortunately, our analysis will show that the unknown MCM can cause severe false peaks.

For accuracy of expression, we consider the point in a spectrum as a peak if and only if its value goes to infinity in the noiseless case, since the peaks, whose values are upper bounded, will not affect the calibration performance when the signal-to-noise ratio (SNR) is large enough. In spectrum $P_{3}(\theta)$, peaks will appear at $\theta$ if and only if

$$
\exists \boldsymbol{v}_{[L]} \neq 0 \text { and } \boldsymbol{U}_{n}^{H} \boldsymbol{Q}_{[L]}(\theta) \boldsymbol{v}_{[L]}=\mathbf{0} .
$$


(C1) $\boldsymbol{Q}_{[L]}(\theta)$ has full column rank but $\boldsymbol{U}_{n}^{H} \boldsymbol{Q}_{[L]}(\theta) \in \mathbb{C}^{(N-M) \times L}$ does not.

(C2) $\boldsymbol{Q}_{[L]}(\theta) \in \mathbb{C}^{N \times L}$ is rank deficient.

To prevent trivial solutions, assume $N \geq M+L$. Then, the peaks caused by condition (C1), which include peaks of real DoAs, depend on $\boldsymbol{U}_{n}$. So the corresponding false peaks, if exist, are noise dependent or unpredictable. While these false peaks are interesting, we want to focus on false peaks caused by condition (C2), which depend only on $\boldsymbol{Q}_{[L]}(\theta)$, and thus are predictable and noise independent. A naive method to identify these peaks would be to calculate the rank of $\boldsymbol{Q}_{[L]}(\theta)$ at each peak. However, this is inefficient and numerically unstable for large $N$. Luckily, Lemma 3.1 shows that the rank of $\boldsymbol{Q}(\theta)$ can be obtained by using its special structure. The proof is given in Appendix A.

Lemma 3.1. Define the set $\Omega_{\beta}=\left\{\theta\left|\theta=\arcsin \left(\frac{k}{N \beta}\right), k \in \mathbb{Z},\right| k \mid \leq \beta N\right\}$. Denote $\Omega_{\beta}^{(o)}$ and $\Omega_{\beta}^{(e)}$ as subsets of $\Omega_{\beta}$ with $k=2 l+1$ and $k=2 l$, respectively, where $l \in \mathbb{Z}$. For $N \geq 3, L \leq N, \theta \in \Omega_{\theta}$, and $n \in \mathbb{Z}, \boldsymbol{Q}(\theta)$ has the following properties:

125 (P1) a necessary condition for (प'2) is $\theta \in \Omega_{\beta}$;

(P2) if $N=2 n+1$, then $\operatorname{rank}\left(\boldsymbol{Q}_{[L]}(\theta)\right)=\min (n+1, L), \forall \theta \in \Omega_{\beta}$;

(P3) if $N=2 n$, then $\operatorname{rank}\left(\boldsymbol{Q}_{[L]}(\theta)\right)=\min (n+1, L), \forall \theta \in \Omega_{\beta}^{(o)}$;

(P4) if $N=2 n$, then $\operatorname{rank}\left(\boldsymbol{Q}_{[L]}(\theta)\right)=\min (n, L), \forall \theta \in \Omega_{\beta}^{(e)}$.

Then sufficient and necessary conditions for predictable peaks are available.

Proposition 3.2. $2^{2}$ Suppose $M+L \leq N, \theta \in \Omega_{\theta}$, and $n \in \mathbb{Z}$. Then condition (d2) induces false peaks in spectrum $P_{3}(\theta)$ if and only if one of the following conditions is satisfied

(C3) $N=2 n+1, L>n+1$, and $\theta \in \Omega_{\beta}$,

(C4) $N=2 n, L>n+1$, and $\theta \in \Omega_{\beta}$,

${ }_{135}(C 5) \quad N=2 n, L=n+1$, and $\theta \in \Omega_{\beta}^{(e)}$.

Proposition 3.2 implies that choosing $L$ can be a trade-off between MCM modeling errors and false peaks. In particular, too small an $L$ will induce severe

\footnotetext{
${ }^{2}$ This result was presented in part in the conference paper [22], where we discussed the "only if" direction.
} 
modeling errors, while too large an $L$ may cause severe false peaks. A reasonable bound for truncating is $L \leq\left\lceil\frac{N}{2}\right\rceil$, such that false peaks induced by condition (C2) are avoided. Interestingly, the choices of $L$ are upper bounded by this value in almost all existing literature for ULA self-calibration. Here, a possible reason based on critical analysis is given, albeit only for subspace methods.

\section{False Peak Analysis in Multi-Dimensional Spectrum}

As the application of a one-dimensional spectrum is limited by the necessary condition $L+M \leq N$, here we study the self-calibration process based on the multidimensional spectra, where the necessary condition can be relaxed.

\subsection{A Dimension-Scalable Spectrum}

Since the workload of finding the maximum of spectrum $P_{2}(\boldsymbol{\theta})$ grows exponentially with $M$, we simplify the spectrum to a dimension scalable one:

$$
P_{4}\left(\boldsymbol{\theta}_{[K]}\right)=1 / \lambda_{1}\left(\left[\boldsymbol{W}\left(\boldsymbol{\theta}_{[K]}\right)\right]^{H} \boldsymbol{W}\left(\boldsymbol{\theta}_{[K]}\right)\right),
$$

where $K(\leq M)$ is an integer, $\boldsymbol{\theta}_{[K]}=\left[\theta_{1}, \ldots, \theta_{K}\right]^{T}$, and

$$
\boldsymbol{W}\left(\boldsymbol{\theta}_{[K]}\right)=\left[\boldsymbol{Q}_{[L]}^{H}\left(\theta_{1}\right) \boldsymbol{U}_{n}, \ldots, \boldsymbol{Q}_{[L]}^{H}\left(\theta_{K}\right) \boldsymbol{U}_{n}\right]^{H} \in \mathbb{C}^{K N \times L} .
$$

This dimension scalable spectrum has been proposed in 23 for array gain calibration, but our problem is more difficult, as the unknowns of MCM may incur severe false peaks. Our aim is to identify these false peaks.

According to $(9)$, a peak appears at $\boldsymbol{\theta}_{[K]}$ in $P_{4}\left(\boldsymbol{\theta}_{[K]}\right)$ if and only if $\exists \boldsymbol{v}_{[L]} \neq \mathbf{0}$ such that $\boldsymbol{U}_{n}^{H} \boldsymbol{Q}_{[L]}\left(\theta_{l}\right) \boldsymbol{v}_{[L]}=0, \forall l \in[K]$, which is further equivalent to

$$
\exists \boldsymbol{v}_{[L]} \neq \mathbf{0} \text { such that } \boldsymbol{H}_{E}\left(\boldsymbol{\theta}_{[K]}\right) \boldsymbol{h}=\mathbf{0},
$$

where $\boldsymbol{h}=\left[\boldsymbol{v}_{[L]}^{T}, \boldsymbol{k}\right]^{T}, \boldsymbol{k}=\left[\boldsymbol{k}_{1}^{T}, \ldots, \boldsymbol{k}_{K}^{T}\right]^{T}$, " $\otimes$ " is the Kronecker product, and

$$
\begin{aligned}
\boldsymbol{H}_{E}\left(\boldsymbol{\theta}_{[K]}\right) & =\left[\boldsymbol{H}\left(\boldsymbol{\theta}_{[K]}\right), \boldsymbol{I}_{K} \otimes \boldsymbol{C A}\right], \\
\boldsymbol{H}\left(\boldsymbol{\theta}_{[K]}\right) & =\left[\boldsymbol{Q}_{[L]}^{T}\left(\theta_{1}\right), \ldots, \boldsymbol{Q}_{[L]}^{T}\left(\theta_{K}\right)\right]^{T} \in \mathbb{C}^{K N \times L},
\end{aligned}
$$

Assume $N \geq L / K+M$ to avoid trivial solutions in 10 . Since $\boldsymbol{I}_{K} \otimes \boldsymbol{C A}$ is full rank, condition 10 holds if and only if any of the two conditions is satisfied 
(C6) $\boldsymbol{k}=\mathbf{0}, \exists \boldsymbol{v}_{[L]} \neq \mathbf{0}$ such that $\boldsymbol{H}_{E}\left(\boldsymbol{\theta}_{[K]}\right) \boldsymbol{h}=\mathbf{0}$ (or $\boldsymbol{H}\left(\boldsymbol{\theta}_{[K]}\right)$ is rank deficient).

(C7) $\exists \boldsymbol{k} \neq \mathbf{0}$ and $\boldsymbol{v}_{[L]} \neq 0$ such that $\boldsymbol{H}_{E}\left(\boldsymbol{\theta}_{[K]}\right) \boldsymbol{h}=\mathbf{0}$.

155 Condition ( $\mathrm{C} 6$ ) only depends on the structure of MCM, and thus the corresponding peaks are predictable, while condition $(\mathrm{C} 7)$ may cause both unpredictable and noise dependent peaks. Next, we will analyze conditions of predictable peaks in both cases. For ease of expression, we will first analyze these false peaks with $K=2$, and then extend the discussion to the $K>2$ case.

To find out false peaks satisfying ( $(\sqrt{6})$, we define the set $\Omega_{\beta}^{2}=\left\{\boldsymbol{\theta}_{[2]} \mid \theta_{m} \in\right.$ $\left.\Omega_{\beta}, m \in[2], \sin \left(\theta_{2}\right)-\sin \left(\theta_{1}\right)=\frac{2 k}{N \beta}, k \in \mathbb{Z}\right\}$ and then introduce the following properties of $\boldsymbol{H}\left(\boldsymbol{\theta}_{[2]}\right)$. Formal arguments are given in Appendix B.

Lemma 4.1. When $L \leq N, N \geq 3$, and $\theta_{m} \in \Omega_{\theta}, \forall m \in[2], \boldsymbol{H}\left(\boldsymbol{\theta}_{[2]}\right)$ has the 165 following properties:

(P5) A necessary condition for ( $(6)$ is $\boldsymbol{\theta}_{[2]} \in \Omega_{\beta}^{2}$;

(P6) Denote $r_{H}=\operatorname{rank}\left(\left[\boldsymbol{Q}_{[L]}^{T}\left(\theta_{1}\right), \boldsymbol{a}_{[L]}\left(\theta_{2}\right)\right]\right)$, then $\operatorname{rank}\left(\boldsymbol{H}\left(\boldsymbol{\theta}_{[2]}\right)\right)=\min \left(r_{H}, L\right)$, $\forall \boldsymbol{\theta}_{[2]} \in \Omega_{\beta}^{2}$, where $r_{H}=\operatorname{rank}\left(\boldsymbol{Q}_{[L]}\left(\theta_{1}\right)\right)$ if $\boldsymbol{a}_{[L]}\left(\theta_{2}\right)$ is in the column space of $\boldsymbol{Q}_{[L]}^{T}\left(\theta_{1}\right)$; otherwise, $r_{H}=\operatorname{rank}\left(\boldsymbol{Q}_{[L]}\left(\theta_{1}\right)\right)+1$.

Then necessary and sufficient conditions for peaks of $(\mathrm{C} 6)$ can be obtained.

Proposition 4.2. When $L \leq N, L \leq 2 N-2 M$, and $\theta_{m} \in \Omega_{\theta}, \forall m \in[2]$, condition (46) induces peaks in spectrum $P_{4}\left(\boldsymbol{\theta}_{[2]}\right)$ if and only if

(C8) $\boldsymbol{\theta}_{[2]} \in \Omega_{\beta}^{2}$ and $L>\operatorname{rank}\left(\boldsymbol{H}\left(\boldsymbol{\theta}_{[2]}\right)\right.$, where $\operatorname{rank}\left(\boldsymbol{H}\left(\boldsymbol{\theta}_{[2]}\right)\right.$ is obtained by (F[G).

To analyze the predictable false peaks satisfying condition (C7), we define the set $\Delta_{\beta}^{2}=\left\{\boldsymbol{\theta}_{[2]} \mid \theta_{m} \in \Omega_{\theta}, m \in[2], \sin \left(\theta_{1}\right)-\sin \left(\theta_{2}\right)=\frac{2 k}{N \beta}, k \in \mathbb{Z}\right\}$ and have the results in Lemma 4.3 , whose proof is given in Appendix C.

Lemma 4.3. Given $L \leq N, L \leq 2 N-2 M, \theta_{m} \in \Omega_{\theta}, \forall m \in[2]$, and $\theta_{1} \neq \theta_{2}$. If $\boldsymbol{\theta}_{[2]} \in \Delta_{\beta}^{2}$, then $\operatorname{rank}\left(\boldsymbol{H}_{E}\left(\boldsymbol{\theta}_{[2]}\right)\right) \leq N+M+3$.

Then, a sufficient condition for predictable peaks caused by $(\mathrm{C} 7 \mathrm{f}$ is obtained. 
Proposition 4.4. Given $L \leq N, L \leq 2 N-2 M, \theta_{m} \in \Omega_{\theta}, \forall m \in[2]$, and $\theta_{1} \neq \theta_{2}$. Condition ( $(\sqrt{7})$ induces peaks in spectrum $P_{4}\left(\boldsymbol{\theta}_{[2]}\right)$ if (C9) $\boldsymbol{\theta}_{[2]} \in \Delta_{\beta}^{2}$ and $L+M>N+3$.

Note that the number of false peaks in Proposition 4.4 is infinite, and thus this will severely contaminate spectrum $P_{4}\left(\boldsymbol{\theta}_{[K]}\right)$.

Let us denote $\mathcal{J}=\left\{\mathcal{K}_{0}, \mathcal{K}_{1}, \ldots, \mathcal{K}_{J}\right\}$ as a partition of the index set $[K]$ and define sets of $\boldsymbol{\theta}_{[K]}$ in Table 1] where $\mathcal{K}_{j}$ is a set of indices with size $K_{j}$ and $\sum_{j=1}^{J} K_{j}=K$. Then, Lemma 4.1 can be extended to the $K>2$ case.

Table 1: A List Of Sets Of $\boldsymbol{\theta}_{[K]}$

\begin{tabular}{l}
$\Omega_{\beta}^{K}=p\left\{\boldsymbol{\theta}_{[K]} \mid \theta_{k} \in \Omega_{\beta}, \sin \left(\theta_{k}\right)-\sin \left(\theta_{1}\right)=\frac{2 i_{k 1}}{N \beta}, i_{k 1} \in \mathbb{Z}\right.$, forallk $\left.\in[K]\right\}$ \\
\hline$\Delta_{\beta}^{K}=\left\{\boldsymbol{\theta}_{[K]} \mid \theta_{k} \in \Omega_{\theta}, \sin \left(\theta_{k}\right)-\sin \left(\theta_{1}\right)=\frac{2 i_{k 1}}{N \beta}, i_{k 1} \in \mathbb{Z}, \forall k \in[K]\right\}$ \\
\hline$\tilde{\Delta}_{\beta, \mathcal{J}}^{K}=\left\{\boldsymbol{\theta}_{[K]} \mid \boldsymbol{\theta}_{\mathcal{K}_{j}} \in \Delta_{\beta}^{K_{j}}, \forall j \in[J],\left(\theta_{k}, \theta_{l}\right) \notin \Delta_{\beta}^{2}, \forall k, l \in \mathcal{K}_{0}, k \neq l\right\}$ \\
\hline$\Delta_{\beta, \mathcal{J}}^{K}=\left\{\boldsymbol{\theta}_{[K]} \mid \boldsymbol{\theta}_{[K]} \in \tilde{\Delta}_{\beta, \mathcal{J}}^{K},\left(\theta_{k}, \theta_{l}\right) \notin \Delta_{\beta}^{2}, \forall k \in \mathcal{K}_{p}, \forall l \in \mathcal{K}_{q}, p \neq q\right\}$
\end{tabular}

Lemma 4.5. When $L \leq N, 3 \leq N, K<N$, and $\theta_{m} \in \Omega_{\theta}, \forall m \in[K], \boldsymbol{H}\left(\boldsymbol{\theta}_{[K]}\right)$ has the following properties: 1. a necessary condition for ( (46) is $\boldsymbol{\theta}_{[K]} \in \Omega_{\beta}^{K}$; 2. Let $r_{H}=\operatorname{rank}\left(\left[\boldsymbol{Q}_{[L]}^{T}\left(\theta_{1}\right), \boldsymbol{a}_{[L]}\left(\theta_{2}\right), \ldots, \boldsymbol{a}_{[L]}\left(\theta_{K}\right)\right]\right)$, then

$$
\operatorname{rank}\left(\boldsymbol{H}\left(\boldsymbol{\theta}_{[K]}\right)\right)=\min \left(r_{H}, L\right), \quad \forall \boldsymbol{\theta}_{[K]} \in \Omega_{\beta}^{K} .
$$

Proof of Lemma 4.5 is similar to that of Lemma 4.1 and omitted. Similarly, predictable peaks satisfying condition ( $\mathrm{C} 6$ ) can be obtained in proposition 4.6

Proposition 4.6. When $L \leq N, K \leq M \leq N, L \leq K(N-M)$, and $\theta_{m} \in \Omega_{\theta}$, $\forall m \in[K]$, condition (C6) induces peaks in spectrum $P_{4}\left(\boldsymbol{\theta}_{[K]}\right)$ if and only if (C10) $\boldsymbol{\theta}_{[K]} \in \Omega_{\beta}^{K}$ and $L>\operatorname{rank}\left(\boldsymbol{H}\left(\boldsymbol{\theta}_{[K]}\right)\right)$ with $\operatorname{rank}\left(\boldsymbol{H}\left(\boldsymbol{\theta}_{[K]}\right)\right)$ given by 12] Next, Lemma 4.7 extends Lemma 4.3 to the $K>2$ case. 
Lemma 4.7. Given $L \leq N, K \leq M, L \leq K(N-M), \theta_{k} \in \Omega_{\theta}, \forall k \in[K]$, and $\theta_{k} \neq \theta_{l}, \forall k \neq l$. If $\boldsymbol{\theta}_{[K]} \in \tilde{\Delta}_{\beta, \mathcal{J}}^{K}$, then $\operatorname{rank}\left(\boldsymbol{H}_{E}\left(\boldsymbol{\theta}_{[K]}\right)\right) \leq r(N, M, \mathcal{J})$, where

$$
r(N, M, \mathcal{J})=K+\left(K_{0}+J\right)(N-1)+\sum_{j=1}^{J} \operatorname{rank}\left(\boldsymbol{D}^{\left(K_{j}\right)}\right),
$$

\section{Self-Calibration Methods}

\subsection{Methods Based on One-Dimensional Spectrum}

According to the analysis in Section 3 , false peaks will appear in the spectrum $P_{3}(\theta)$ when $L>\left\lceil\frac{N}{2}\right\rceil$. To avoid them, a naive method is to ignore the peaks satisfying Proposition 3.2 . However, a peak of true DoA will also be missed if it happens to satisfy the proposition. We thus propose a normalized spectrum

$$
P_{3 N}(\theta)=\max _{\boldsymbol{v}_{[L]}, v_{1}=1}\left\|\boldsymbol{Q}_{[L]}(\theta) \boldsymbol{v}_{[L]}\right\|^{2} /\left\|\boldsymbol{W}(\theta) \boldsymbol{v}_{[L]}\right\|^{2} .
$$

According to Lemma $3.1, \boldsymbol{Q}_{[L]}^{H}(\theta) \boldsymbol{Q}_{[L]}(\theta)$ is positive-definite $\forall \theta \notin \Omega_{\beta}$ and there210

fore the optimal $\boldsymbol{v}_{[L]}$ is the eigenvector corresponding to the minimum generalized eigenvalue of the matrix pencil $\left(\boldsymbol{W}^{H} \boldsymbol{W}, \boldsymbol{Q}_{[L]}^{H} \boldsymbol{Q}_{[L]}\right)\left[24\right.$. If $\theta \in \Omega_{\beta}, P_{3 N}(\theta)$ 
in (14) is not well defined. Fortunately, it can be defined by the optimal objective function value of problem (D.1), which can be solved by interior point methods [25. The virtues of spectrum $P_{3 N}(\theta)$ are listed in Proposition 5.1. which is proved in Appendix D.

Proposition 5.1. Assume that $N \geq M+L$ and the number of snapshot $N_{T}$ is large enough, then spectrum $P_{3 N}(\theta)$ has the following properties:

(P7) False peaks in Proposition 3.2, i.e., caused by $\boldsymbol{Q}_{[L]}(\theta) \boldsymbol{v}_{[L]}=\mathbf{0}$, are avoided; (P8) Peaks of true DoA in $P_{3}(\theta)$ will not be missed in $P_{3 N}(\theta)$;

(P9) If the optimal solution of problem (14), i.e., $\boldsymbol{v}_{[L]}$, is uniqu $\}^{3}$ at the peak of a true DoA, then it is a complex scaled version of $\boldsymbol{c}_{[L]}$.

The normalized spectrum can be applied directly in algorithms based on $P_{3}(\theta)$, such as RARE [16, 17] or R-RARE [19], by replacing $P_{3}(\theta)$ with $P_{3 N}(\theta)$. We here propose a new method in Algorithm 1, where the $T$ largest peaks in the spectrum $P_{3 N}(\theta)$ are investigated, and the corresponding estimates of MCM and DoAs, i.e., $\boldsymbol{V}_{t}$ and $\boldsymbol{\theta}_{t}, \forall t \in[T]$, are calculated. The estimation of $\boldsymbol{V}_{t}$ in Steps 4 is based on the property (P 9 ). While the estimation of DoAs $\boldsymbol{\theta}_{t}$ in Step 5 is based on the fact that the spectrum is actually the MUSIC type if $\boldsymbol{V}_{t}=\boldsymbol{C}$. In Step 7, the final estimate is determined by a normalized multidimensional 230 $\quad$ spectrum $\frac{\left\|\boldsymbol{H}(\boldsymbol{\theta}) \boldsymbol{v}_{[L]}\right\|^{2}}{\left\|\boldsymbol{W}(\boldsymbol{\theta}) \boldsymbol{v}_{[L]}\right\|^{2}}$, which is maximized when $\boldsymbol{\theta}=\tilde{\boldsymbol{\theta}}$ and $\boldsymbol{v}_{[L]}=\boldsymbol{c}_{[L]}$.

The reasons for investigating $T>1$ peaks are twofold. First, when the SNR is small, peaks of true DoA may be buried within spectrum fluctuations caused by noise. Second, if the maximum peak happens to be in $\Omega_{\beta}$, then the corresponding $\boldsymbol{Q}_{[L]}(\theta)$ is rank deficient and the optimal solution of $\boldsymbol{v}_{[L]}$ is not unique. In this case, a false calibration will be obtained if $T=1$.

\subsection{Methods Based on Multi-Dimensional Spectrum}

To suppress peaks satisfying $(\mathrm{C} 10)$, we extend spectrum $P_{3 N}(\theta)$ to

$$
P_{4 N}\left(\boldsymbol{\theta}_{[K]}\right)=\max _{\boldsymbol{v}_{[L]}}\left(\left\|\boldsymbol{H}\left(\boldsymbol{\theta}_{[K]}\right) \boldsymbol{v}_{[L]}\right\|^{2} /\left\|\boldsymbol{W}\left(\boldsymbol{\theta}_{[K]}\right) \boldsymbol{v}_{[L]}\right\|^{2}\right) .
$$

\footnotetext{
${ }^{3}$ The optimal solution of $\sqrt{\mathrm{D} .1}$ is not unique when $\theta \in \Omega_{\beta}$.
} 


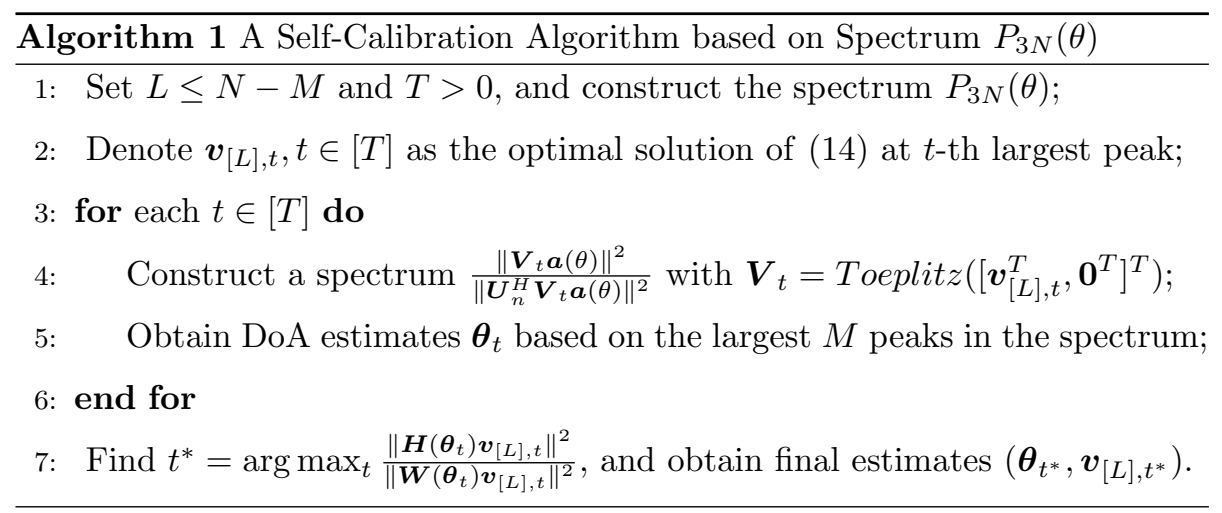

and introduce a penalty parameter $d\left(\boldsymbol{\theta}_{[K]}\right)$ for false peaks in (C11) and obtain

$$
P_{5}\left(\boldsymbol{\theta}_{[K]}\right)=d\left(\boldsymbol{\theta}_{[K]}\right) P_{4 N}\left(\boldsymbol{\theta}_{[K]}\right) .
$$

We design $d\left(\boldsymbol{\theta}_{[K]}\right)$ in two steps. To suppress peaks caused by $\theta_{k}=\theta_{l}$, set

$$
d\left(\boldsymbol{\theta}_{[K]}\right)=\tilde{d}_{c 13}=\min _{k, l \in[K], k \neq l}\left|b_{k}-b_{l}\right|^{2},
$$

where $b_{k}=e^{j \pi \beta \sin \left(\theta_{k}\right)}$. Note that $\tilde{d}_{c 13}$ may harm the array resolution capability, since a desirable peak of true DoAs may be diminished if two of the DoAs are close to each other. To reduce this risk, we modify (17) to

$$
d\left(\boldsymbol{\theta}_{[K]}\right)=d_{c 13}=\min \left(\tilde{d}_{c 13} / \epsilon_{e}, 1\right)
$$

where $\epsilon_{e}$ is a small positive constant. Then the peak will not be diminished unless $\tilde{d}_{c 13}<\epsilon_{e}$. Second, to suppress the false peaks satisfying ( 111 , i.e., in set $\tilde{\Delta}_{\beta, \mathcal{J}}^{K}$ whenever $r(N, M, \mathcal{J})<L+K M$, we define

$$
d\left(\boldsymbol{\theta}_{[K]}\right)=\tilde{d}_{\mathcal{J}, K}=\sum_{j=1}^{J}\left(\max _{k, l \in \mathcal{K}_{j}}\left|b_{k}^{N}-b_{l}^{N}\right|^{2}\right) .
$$

It is seen that $\tilde{d}_{\mathcal{J}, K}=0$ if and only if a point is in a set $\tilde{\Delta}_{\beta, \mathcal{J}}^{K}$. Hence, the values of $P_{4 N}\left(\boldsymbol{\theta}_{[K]}\right)$ will be suppressed to 0 for all $\boldsymbol{\theta}_{[K]}$ in $\tilde{\Delta}_{\beta, \mathcal{J}}^{K}$.

In (19), for a given tuple $(N, M, L, K)$, the partition $\mathcal{J}$ satisfying $r(N, M, \mathcal{J})<$ $L+K M$ is generally not unique. To suppress all corresponding false peaks, we denote the set of all satisfied partitions by $\tilde{\mathcal{J}}$ and define

$$
d\left(\boldsymbol{\theta}_{[K]}\right)=\tilde{d}_{\underline{11}}=\min _{\mathcal{J} \in \tilde{\mathcal{J}}} \tilde{d}_{\mathcal{J}, K}
$$


Although listing all elements of $\tilde{\mathcal{J}}$ is difficult when $K$ is large, a small $K$ is always preferred in practice. In Table 2 , we list all possible $\mathcal{J}, r(N, M, \mathcal{J})$, and $\tilde{d}_{111}$ for $K=2$ and $K=3$, respectively. In the table, $\mathcal{J}_{k}=\left\{\mathcal{K}_{0}, \mathcal{K}_{1}\right\}, \forall k \in[3]$, where $\mathcal{K}_{0}=\{k\}$ and $\mathcal{K}_{1}$ contains the rest two elements in set [3].

\begin{tabular}{llll}
\multicolumn{4}{c}{ Table 2: A List of $\tilde{d}_{\llbracket 11}$ with $K=2$ and $K=3$} \\
\hline$K$ & $\mathcal{J}$ & $\tilde{d}_{\text {[11 }}$ & $r(N, M, \mathcal{J})$ by $[13$ \\
\hline 2 & {$[2]$} & $\left|b_{2}^{N}-b_{1}^{N}\right|^{2}$ & $N+M+3$ \\
3 & {$[3]$} & $\max _{m, n \in[3]}\left|b_{m}^{N}-b_{n}^{N}\right|^{2}$ & $r(N, M,[3])$ \\
3 & $\mathcal{J}_{k}$ & $\min _{m, n \in[3], m \neq n}\left|b_{m}^{N}-b_{n}^{N}\right|^{2}$ & $r\left(N, M, \mathcal{J}_{k}\right)$ \\
\hline
\end{tabular}

On the other hand, $\tilde{d}_{11}$ may suppress a desired peak of true DoAs, if it happens to locate nearby $\tilde{\Delta}_{\beta, \mathcal{J}}^{K}$. To reduce this risk, we modify 20 to

$$
d\left(\boldsymbol{\theta}_{[K]}\right)=d_{\underline{11}}=\min \left(\tilde{d}_{\llbracket 11} / \epsilon_{b}, 1\right),
$$

and apply it only if $r(N, M, \mathcal{J})<K M+L+r_{0}$, where $\epsilon_{b}$ is a small positive constant and $r_{0}$ is a parameter to tradeoff between two risks. Since $r(N, M, \mathcal{J}) \geq$ $\operatorname{rank}\left(\boldsymbol{H}_{E}\left(\boldsymbol{\theta}_{[K]}\right)\right), \boldsymbol{H}_{E}\left(\boldsymbol{\theta}_{[K]}\right)$ may be rank deficient even if $r(N, M, \mathcal{J}) \geq K M+L$, and thus induce false peaks in $\tilde{\Delta}_{\beta, \mathcal{J}}^{K}$. Therefore, a larger $r_{0}$ means a lower risk of detecting a false peak but a higher risk of missing a desired peak, and vice versa. Since the probability of all desired peaks are missed is small, we choose $r_{0}=2$ heuristically when $K \leq 3$.

Combining equations 18 and 21 , we obtain

$$
d\left(\boldsymbol{\theta}_{[K]}\right)=\left\{\begin{array}{lr}
d_{c 13}, & \text { if } \nexists \mathcal{J} \text { satisfying } r(N, M, \mathcal{J}) \geq L+K M+r_{0} ; \\
d_{\llbracket 11,} & \text { otherwise. }
\end{array}\right.
$$

\subsection{Some Remarks on Algorithm Implementation}

\subsubsection{Performance and complexity trade-off}

In Algorithm 2, the complexity of exhaustively searching the spectrum is proportional to $G^{K}$, where $G$ is the size of uniform grid used to cover the obser- 


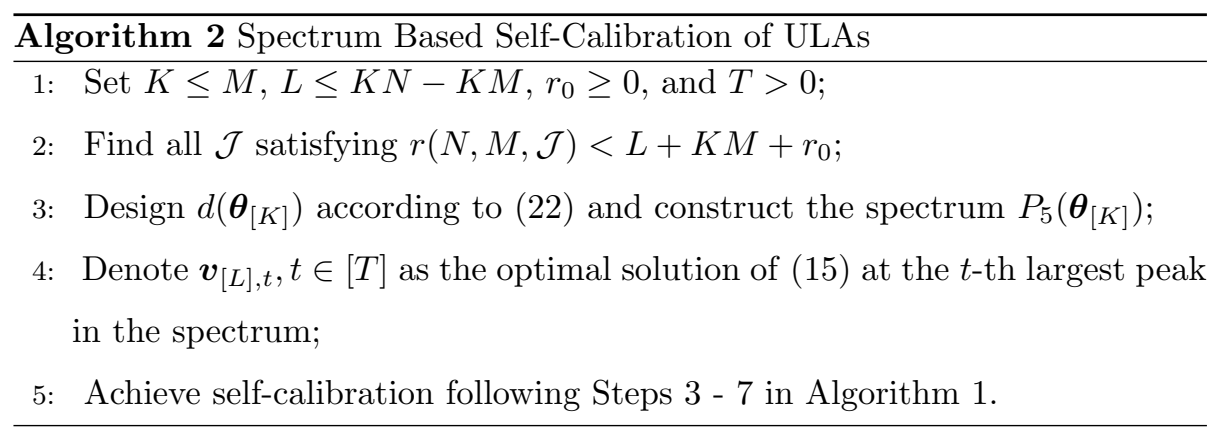

vation area, e.g., $\left[-\frac{\pi}{2}, \frac{\pi}{2}\right]$. Clearly, too dense a grid will cause a high workload, while a coarse grid may impact accuracy of calibration. For an efficient tradeoff, $K$ should be chosen as small as possible.

\subsubsection{Some unpredictable false peaks}

The false peaks in Sections 3 and 4 are predicable. On the other hand, some peaks may be caused by special patterns of real DoAs and satisfy condition (C1), such as those listed in Proposition 5.2. These peaks are unpredictable.

Proposition 5.2. Denote $\boldsymbol{\theta}_{[2]}=\left[\theta_{1}, \theta_{2}\right]^{T}$ and two of the true DoAs as $\left(\tilde{\theta}_{1}, \tilde{\theta}_{2}\right)$.

1. If $\tilde{\theta}_{1}=-\tilde{\theta}_{2}$ and $L>\frac{N-1}{2}$, a false peak appears at $P_{3}(0)$.

2. If $\tilde{\theta}_{1}=-\tilde{\theta}_{2}$ and $L \geq N-1$, false peaks appear in $P_{4}\left(\boldsymbol{\theta}_{[2]}\right)$ when $\theta_{1}=-\theta_{2}$.

3. If $\tilde{\theta}_{1}=0$ and $L=N$, false peaks appear in $P_{4}\left(\boldsymbol{\theta}_{[2]}\right)$ when $\theta_{1}=-\theta_{2}$.

Proof. The lemma can be derived by using the equations $\boldsymbol{C}=\boldsymbol{J}_{N} \boldsymbol{C} \boldsymbol{J}_{N}, \boldsymbol{a}(-\theta)=$ $\boldsymbol{J}_{N} \boldsymbol{a}(\theta) t(\theta)$, and $\boldsymbol{C a}(-\theta)=t(\theta) \boldsymbol{J}_{N} \boldsymbol{C a}(\theta)$, where $t(\theta)=e^{j(N-1) \pi \beta \sin (-\theta)}$.

\section{Numerical Examples}

The simulations are based on ULAs under three different settings: $N=7$, 270 $N=9$, and $N=16$ with mutual coupling coefficients denoted by $\boldsymbol{c}_{1}, \boldsymbol{c}_{2}$, and $\boldsymbol{c}_{3}{ }^{4}$ respectively. For a given $L$, observations are generated with $\boldsymbol{c}_{i}(l)=0, \forall l>L$.

\footnotetext{
${ }^{4} \boldsymbol{c}_{1}$ follows from the settings in [20] $. \boldsymbol{c}_{2}=[1,0.0459-0.428 i,-0.1157+0.2134 i, 0.1168-$ $0.1185 i,-0.1079+0.0655 i, 0.0965-0.031 i,-0.0844+0.0074 i, 0.072+0.0098 i,-0.0597-0.0219 i]$ and $\boldsymbol{c}_{3}=[1,0.0454-0.4282 i,-0.1154+0.2136 i, 0.1167-0.1187 i,-0.1079+0.0656 i, 0.0964-$ $0.0311 i,-0.0844+0.0075 i, 0.072+0.0097 i,-0.0598-0.0219 i,-0.0253+0.0602 i,-0.0363-$
} 

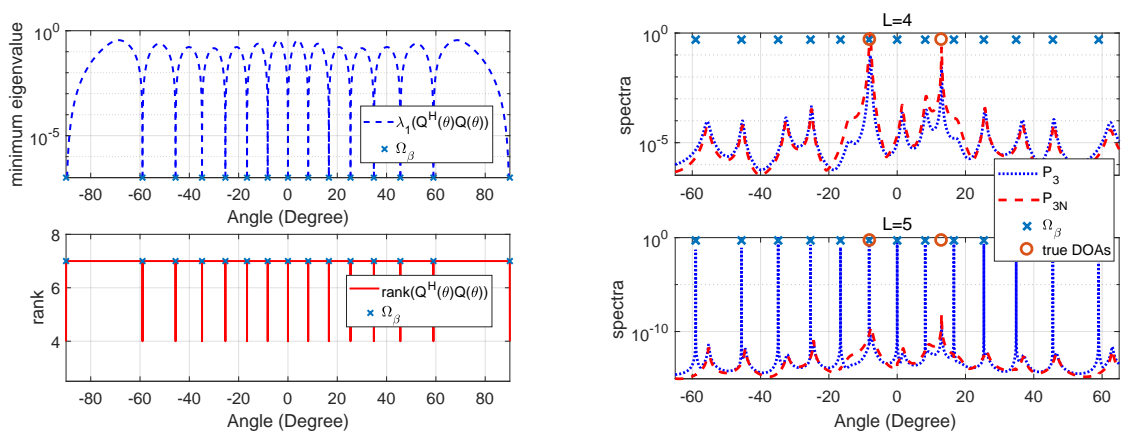

Figure 1: The minimum eigenvalue and rank of $\boldsymbol{Q}^{H}(\theta) \boldsymbol{Q}(\theta)$ versus $\theta$ with $L=N$.

Figure 2: Spectra $P_{3}(\theta)$ and $P_{3 N}(\theta)$ with different $L, \mathrm{SNR}=30 \mathrm{~dB}$, and $N_{T}=100$.

\subsection{Validity of Theoretical Analysis}

Lemma 3.1: Figure 1 shows the minimum eigenvalues and ranks of $\boldsymbol{Q}^{H}(\theta) \boldsymbol{Q}(\theta)$ varying with the $\operatorname{DoA} \theta$, when $N=7$. It is seen that $\boldsymbol{Q}(\theta)$ is rank deficient if and only if $\theta \in \Omega_{\beta}$, and the rank of $\boldsymbol{Q}_{[L]}(\theta)$ coincides with Lemma 3.1 .

Lemma 3.2. We set $N=7$, the true DoAs $\tilde{\boldsymbol{\theta}}=\left[-8^{\circ}, 13^{\circ}\right]$, and SNR $=30 \mathrm{~dB}$. The resulting spectra $P_{3}(\theta)$ and $P_{3 N}(\theta)$ are plotted in Fig. 2 with $L=4$ and $L=5$, respectively. We see that DoAs can be detected based on the largest two peaks in spectrum $P_{3 N}(\theta)$ in both situations and spectrum $P_{3}(\theta)$ fails when $L>4$, which coincides with condition (C3) in Proposition 3.2 .

Lemma 4.1: The rank of $\boldsymbol{H}\left(\boldsymbol{\theta}_{[2]}\right)$ with $N=7$ is shown in Fig. 3. It is seen that $\boldsymbol{H}\left(\boldsymbol{\theta}_{[2]}\right)$ is rank deficient only when $\boldsymbol{\theta}_{[2]} \in \Omega_{\beta}^{2}, \operatorname{rank}\left(\boldsymbol{H}\left(\boldsymbol{\theta}_{[2]}\right)\right)=5=$ $\operatorname{rank}\left(\boldsymbol{Q}\left(\theta_{1}\right)\right)+1$ for most points in $\Omega_{\beta}^{2}$, and this value decays to 4 when $\theta_{1}= \pm \theta_{2}$ such that $\boldsymbol{a}\left(\theta_{2}\right)$ is in the column space of $\boldsymbol{Q}^{T}\left(\theta_{1}\right)$.

The first three experiments validate our theoretical analyses in scenarios where $N$ is odd. For even $N$, similar conclusions can be drawn and are omitted.

Lemma 4.3: Consider the 16-element ULA and denote differences between

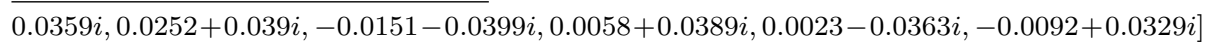
are generated according to electromagnetic theory [2] 

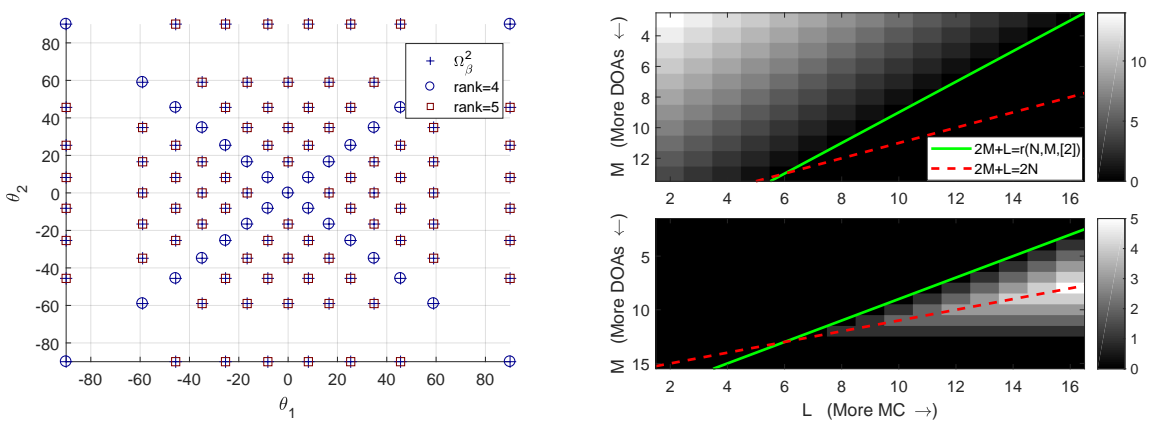

Figure 3: Rank of $\boldsymbol{H}\left(\boldsymbol{\theta}_{[2]}\right), N=7$, and $L=$ Figure 4: $\quad \delta_{1}(16, M, L,[2]) \quad$ (upper) and $N$. Only the points with $\operatorname{rank}\left(\boldsymbol{H}\left(\boldsymbol{\theta}_{[2]}\right)\right)<L \quad \delta_{2}(16, M, L,[2])$ (lower) vary with $M$ and $L$, are marked out. where $N=16, K=2$.

theoretical upper bounds of $\operatorname{rank}\left(\boldsymbol{H}_{E}\left(\boldsymbol{\theta}_{[K]}\right)\right)$ and its numerical value by

$$
\begin{aligned}
& \delta_{1}(N, M, L, \mathcal{J})=r(N, M, \mathcal{J})-r_{\max } \\
& \delta_{2}(N, M, L, \mathcal{J})=\min (K N, K M+L)-r_{\max }
\end{aligned}
$$

where $r_{\text {max }}=\max _{\boldsymbol{\theta}_{[K]} \in \tilde{\Delta}_{\beta, \mathcal{J}}^{K}} \operatorname{rank}\left(\boldsymbol{H}_{E}\left(\boldsymbol{\theta}_{[K]}\right)\right)$. When $K=2, \mathcal{J}=[2]$ and $r(N, M,[2])=N+M+3$. In Fig. 4, we plot $\delta_{1}(N, M, L, \mathcal{J})$ and $\delta_{2}(N, M, L, \mathcal{J})$ for various $L$ and $M$, respectively. The lines, where $r(N, M, \mathcal{J})=K M+L$ and $K N=K M+L$, are also marked out.

At the top of Fig. 44 we observe that $\operatorname{rank}\left(\boldsymbol{H}_{E}\left(\boldsymbol{\theta}_{[2]}\right)\right) \leq r(N, M,[2]), \forall \boldsymbol{\theta}_{[K]} \in$ $\Delta_{\beta}^{2}$, whenever $K N \geq K M+L$ and $M \leq N-3$. This result coincides with Lemma 4.3 As such, in the area between the dashed and solid lines, where $r(N, M,[2])<K M+L$, we can expect that $\boldsymbol{H}_{E}\left(\boldsymbol{\theta}_{[2]}\right)\left(\in \mathbb{C}^{K N \times K M+L}\right)$ is rank deficient for all $\boldsymbol{\theta}_{[K]} \in \Delta_{\beta}^{2}$, which is observed at the bottom of this figure. Moreover, when $\boldsymbol{H}_{E}\left(\boldsymbol{\theta}_{[2]}\right)$ is rank deficient, false peaks will appear according to condition (C7). Hence, condition ( $\mathrm{C} 9 \mathrm{~g}$ in Proposition 4.4 is also verified.

Lemma 4.7 can also be validated in a similar setting and omitted here. 


\subsection{Simulations for the Proposed Algorithms} large. The online method is similarly to MUSIC, since it it initialized by $\boldsymbol{C}=\boldsymbol{I}$. The working area of mid-array is bounded by its necessary condition.

The application ranges of the rest methods can be explained by analytical results in the paper. Overall, they are restricted by the necessary condition and Alg-P4 are limited by $L<9$, which are based on conditions (C4) and (C5) in Proposition 3.2 and (C8) in Proposition 4.2 , respectively. It is interesting to see that blind method may fail when $9 \leq L<11$ or $r(N, M,[2])=2 M+L-1$. The 


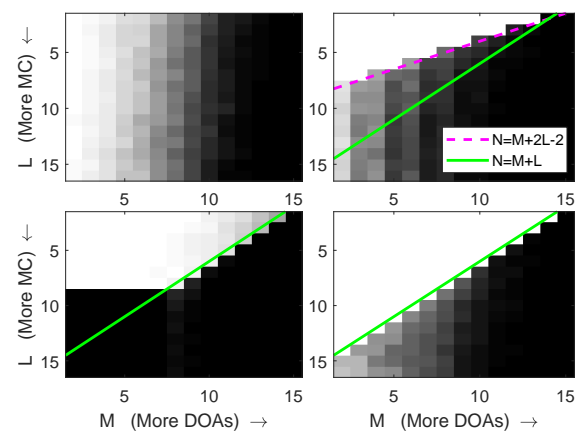

(a)

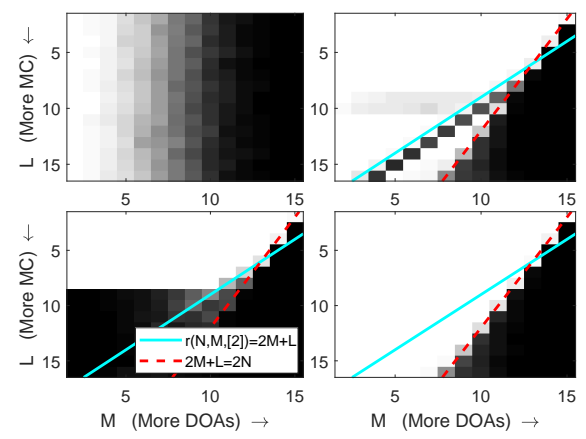

(b)

Figure 5: PoR varies with $M$ and $L$, where white and black colors indicate complete success and complete failure: (a) MUSIC (top left), mid-array (top right), R-RARE (bottom left), Alg-1 (bottom right), (b) online algorithm (top left), blind method (top right), Alg-P4 (bottom left), Alg-2 (bottom right)

reason is that blind method is based on a spectrum $\frac{\lambda_{2}\left(\boldsymbol{W}^{H}\left(\boldsymbol{\theta}_{[2]}\right) \boldsymbol{W}\left(\boldsymbol{\theta}_{[2]}\right)\right)}{\lambda_{1}\left(\boldsymbol{W}^{H}\left(\boldsymbol{\theta}_{[2]}\right) \boldsymbol{W}\left(\boldsymbol{\theta}_{[2]}\right)\right)}$, which can suppress predictable false peaks if $\lambda_{1}$ and $\lambda_{2}$ approach to 0 , simultaneously. However, by utilizing Lemma 4.1 and Lemma 4.3 , it can be easily derived that this property fails when $9 \leq L \leq 11$ or $r(N, M,[2])=2 M+L-1$, respectively.

Alg-1 and Alg-2 apply most broadly. We see that Alg-1 works perfectly when $L+M \leq N$, and the same is true for Alg-2 when $L<\min (2 N-2 M, N-1)$. This implies that the analysis for predictable peaks in spectrum $P_{3}(\theta)$ and $P_{4}\left(\boldsymbol{\theta}_{[2]}\right)$ is exhaustive. If not, the remaining predictable false peaks will cause the PoR to go to 0. Alg-2 may be affected by unpredictable false peaks when $L \geq N-1$ (which agrees with Lemma 5.2) or $2 M+L \geq 2 N$. To the best of our knowledge, truncation of the effect of mutual coupling (i.e., setting $\left.c_{l}=0, \forall l>L\right)$ is required in all existing self-calibration methods. Our analytical results and the corresponding simulation give reasonable bounds for choosing $L$ in these methods. Moreover, truncation is not required in Alg-2 when $M<8$ in the simulation.

Next, we investigate the impact of parameters $\epsilon_{e}$ in 18 and $\epsilon_{b}$ in 21 to Alg-2. Note that $d\left(\boldsymbol{\theta}_{[K]}\right)$ can suppress false peaks, but also induces risks of 


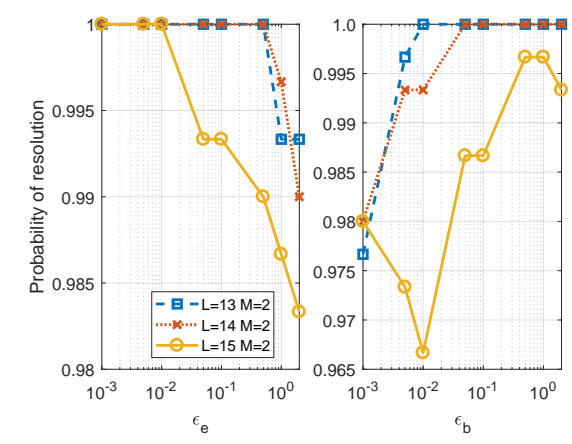

Figure 6: PoR of Alg-2 varies with $\epsilon_{e}$ (left) and $\epsilon_{b}$ (right), where $N=16$ and $S N R=20 \mathrm{~dB}$.

missing peaks of true DoAs. Especially, when $M=K=2$, missing the only peak of true DoAs means a failure of estimation. Hence, $M=2$ with $L$ varying from 13 to 15 are very difficult settings. In Fig. 6, we see that Alg-2 is insensitive to $\epsilon_{e}$ and $\epsilon_{b}$, and $\epsilon_{e}=0.01$ and $\epsilon_{b}=1$ are good choices.

Next, we investigate the asymptotic performance of different algorithms. The results of one-dimensional spectrum based methods and the other methods are illustrated in Fig. $7 \mathrm{a}$ and $7 \mathrm{~b}$, respectively. When $L=4$ and $M=2$, we see that all methods work well. The PoRs of Alg-2, Alg-P4, blind, and online method are always equal to 1 and their curves are omitted. In a more difficult situation, where $L=10$ and $M=5$, we see that Alg- 1 and Alg-2 perform better than the other methods, and the PoR of Alg-2 approaches 1 when $N_{T} \geq 100$. When $L=10$ and $M=10$, only Alg-2 can work.

\subsubsection{Accuracy of self-calibration}

First, consider the 9-element array which is illuminated by $M=3$ sources located at $\tilde{\boldsymbol{\theta}}=\left[-20^{\circ},-1^{\circ}, 5^{\circ}\right]^{T}$. The first two sources are correlated with correlation coefficient [4] $\rho=0.5$. We first compare Alg-1 to existing algorithms with $L=6$, and then Alg-2 to existing algorithms with $L=8$. The simulation results, including PoR and root mean square error (RMSE) of DoA estimation of successful detections, are plotted in Fig. 8 and 9 respectively. In the figures, " $T=t$ " denotes implementing Alg-1 or Alg-2 with $T=t$. 


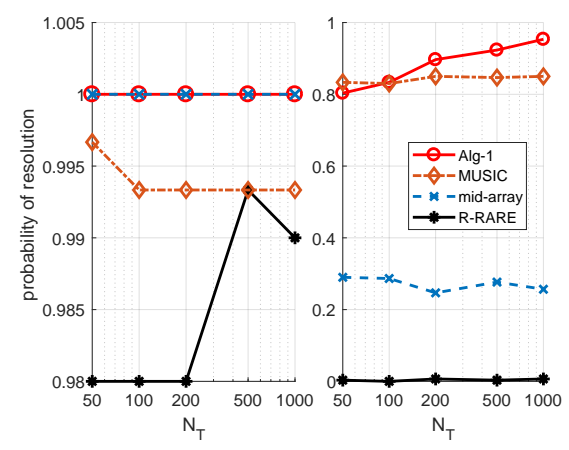

(a)

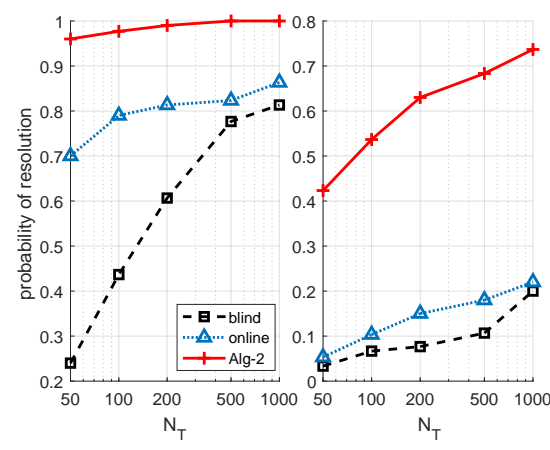

(b)

Figure 7: PoR versus $N_{T}$ with $N=16$ and $S N R=20 \mathrm{~dB}$. (a) Left: $L=4, M=2$; right: $L=10, M=5$; (b) Left: $L=10, M=5$; right: $L=10, M=10$.

In Fig. 8a, we see that Alg-1 may fail in the low SNR region, since it is impacted by unpredictable fluctuations. This phenomenon is alleviated by using a larger $T$ or a higher SNR. R-RARE does not work, since its spectrum is polluted by the false peaks in $\Omega_{\beta}$. The curves of mid-array and online methods are close to 0 and omitted. Online method is for independent sources only [14.

In Fig. 8b, it is seen that RMSE curves of Alg-1 decrease with the increase of SNR, while that of MUSIC is bounded because of modeling mismatch in MCM. The gap between Cramer-Rao bound (CRB) and the RMSE of Alg-1 is caused by the performance loss of subspace method when sources are correlated.

In Fig. 9, we see that Alg-2 and blind method perform well, which coincides with the results in Fig. 5. The floor effect of Alg-2 is caused by a coarse searching grid. The PoR of Alg-P4 and online method are close to 0 and omitted.

Finally, asymptotic performance of different methods are investigated with $N=16$ and $L=10 . \quad M=5$ independent signal sources are equally spaced between $1^{\circ}$ and $16^{\circ}$. The results are shown in Fig. 10 and 11, where curves of methods with PoR approaching zero are omitted and RMSE are based on successful detections. It is seen that only Alg-1, Alg-2, and blind algorithm can work reliably. Their calibration performance improve as $N_{t}$ or $T$ becomes large. The gap between RMSE and CRB can be reduced by substituting the obtained 


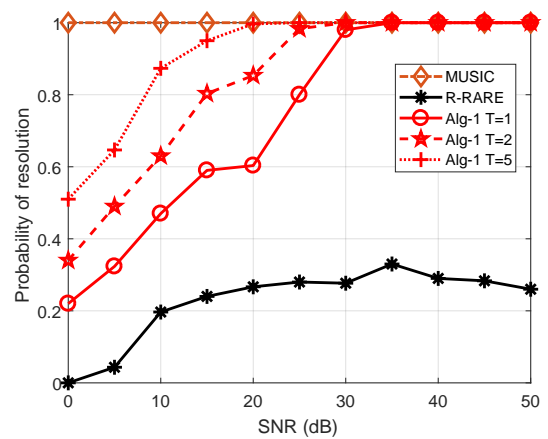

(a)

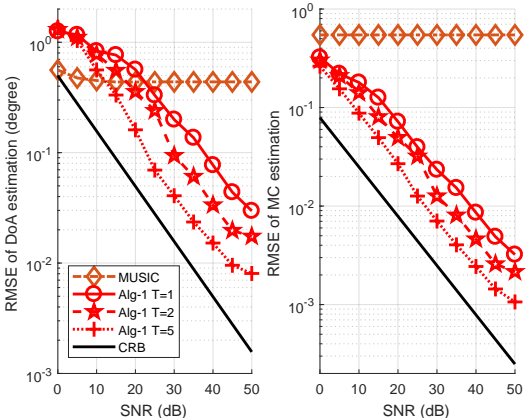

(b)

Figure 8: Calibration performance of different algorithms with $N=9$ and $L=6$ : (a) The PoR versus SNR, (b) RMSE of DoA (left) and mutual coupling (right) estimations of successful resolution versus SNR,

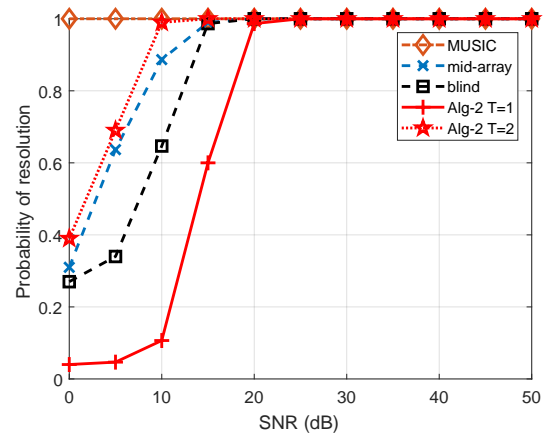

(a)

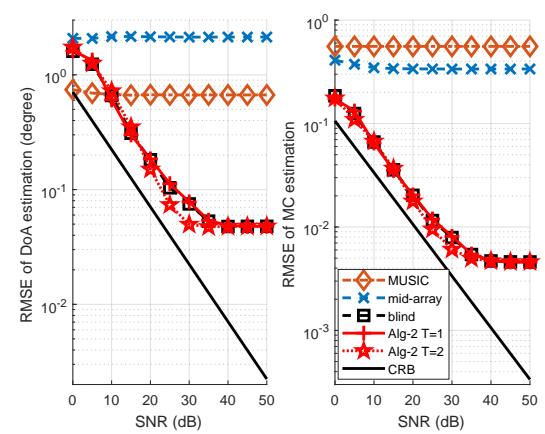

(b)

Figure 9: Calibration performance of different algorithms with $N=9$ and $L=8$ : (a) The PoR versus SNR, (b) RMSE of DoA (left) and mutual coupling (right) estimation of successful resolutions versus SNR.

estimates into a local search scheme, e.g., those proposed in [13] and [14.

\section{Conclusions}

This paper has investigated the effect of mutual coupling to DoA estimation in ULAs. We considered subspace methods and found that the unknown MCM can cause false peaks in their spatial spectra, giving rise to ambiguous DoA 


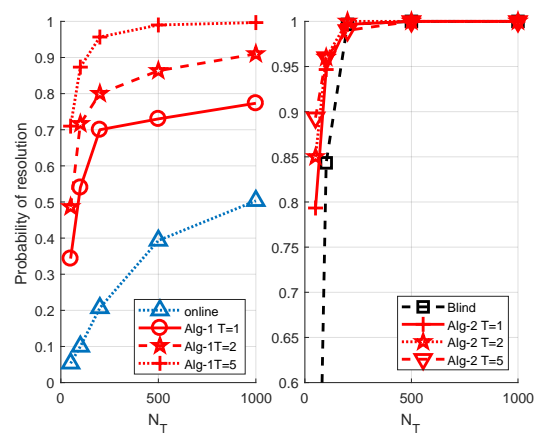

Figure 10: PoR versus $N_{t}$ with $N=16, L=10, M=5$, and $S N R=20 \mathrm{~dB}$.

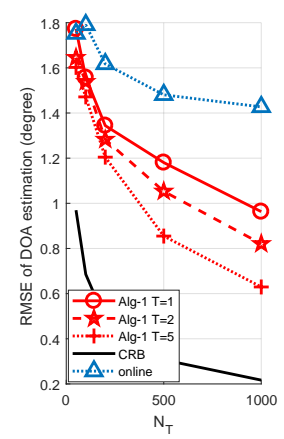

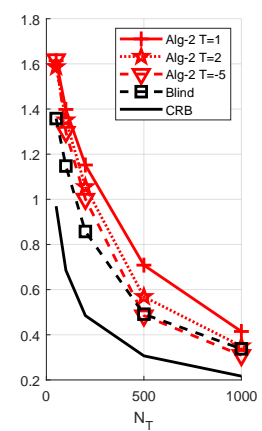

(a)

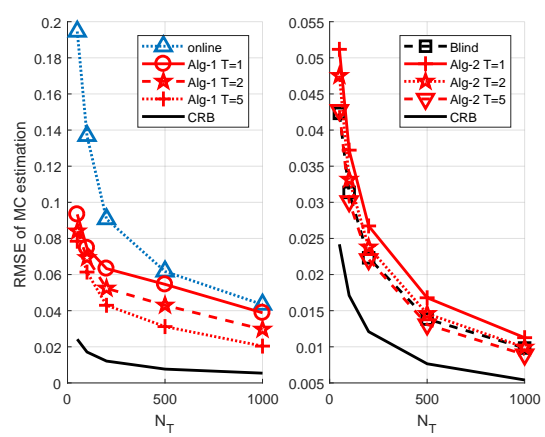

(b)

Figure 11: Accuracy of calibration corresponding to Fig. 10 (a) RMSE of DoA estimation, (b) RMSE of mutual coupling calibration.

estimation. The conditions for these false peaks have been analyzed. By utilizing which, we proposed new spectra and algorithms for self-calibration. Simulation results have shown that proposed algorithms are robust to unknown MCM. 


\section{Appendix A. Proof of Lemma 3.1}

To prove the lemma, we first introduce the following notations and lemmas. Denote $b=e^{j \pi \beta \sin (\theta)}$. Then $\boldsymbol{Q}_{[L]}(b)=\boldsymbol{Q}_{[L]}(\theta)$. Define $\boldsymbol{b}=\left[b^{1}, \ldots, b^{N}\right]^{T}$ and

$$
\begin{aligned}
\boldsymbol{E}_{N}(b) & =\boldsymbol{I}_{N}-b \boldsymbol{J}_{N}, \\
\boldsymbol{F}(b) & =\boldsymbol{I}_{N}-b \boldsymbol{I}_{N}^{(1)} .
\end{aligned}
$$

Obviously, $\boldsymbol{F}(b)$ is a full rank matrix and $|\boldsymbol{F}(b)|=1$.

Lemma Appendix A.1. The eigenvalue decomposition of $\boldsymbol{E}_{N}(b)$ is

$$
\boldsymbol{E}_{N}(b)=\boldsymbol{U}_{E} \boldsymbol{D}_{E} \boldsymbol{U}_{E}^{H}
$$

where $\boldsymbol{D}_{E}=\operatorname{blkdg}\left[(1-b) \boldsymbol{I}_{n},(1+b) \boldsymbol{I}_{n}\right]$ if $N=2 n, \boldsymbol{D}_{E}=\operatorname{blkdg}\left[(1-b) \boldsymbol{I}_{n+1},(1+\right.$ b) $\left.\boldsymbol{I}_{n}\right]$ if $N=2 n+1$, and

$$
\boldsymbol{U}_{E}=\frac{1}{\sqrt{2}}\left[\begin{array}{cc}
\boldsymbol{I}_{n} & -\boldsymbol{J}_{n} \\
\boldsymbol{J}_{n} & \boldsymbol{I}_{n}
\end{array}\right], \text { if } N=2 n ; \boldsymbol{U}_{E}=\frac{1}{\sqrt{2}}\left[\begin{array}{ccc}
\boldsymbol{I}_{n} & \mathbf{0} & -\boldsymbol{J}_{n} \\
\mathbf{0}^{T} & \sqrt{2} & \mathbf{0}^{T} \\
\boldsymbol{J}_{n} & \mathbf{0} & \boldsymbol{I}_{n}
\end{array}\right] \text {, otherwise. }
$$

395

Proof. When $N=2 n$, there are $\boldsymbol{I}_{N}=\boldsymbol{U}_{E} \boldsymbol{U}_{E}^{H}$ and $\boldsymbol{J}_{N}=\boldsymbol{U}_{E} \operatorname{blkdg}\left[\boldsymbol{I}_{n},-\boldsymbol{I}_{n}\right] \boldsymbol{U}_{E}^{H}$. Inserting them into A.1 leads to A.3. The proof is similar for $N=2 n+1$.

Corollary Appendix A.2. If $N$ and $k$ are both odd, $\operatorname{rank}\left[\boldsymbol{E}_{N}\left((-1)^{k}\right)\right]=$ $\left\lfloor\frac{N}{2}\right\rfloor+1 ;$ otherwise, $\operatorname{rank}\left[\boldsymbol{E}_{N}\left((-1)^{k}\right)\right]=\left\lfloor\frac{N}{2}\right\rfloor$.

Then we prove the first two properties of Lemma 3.1. The rest can be derived similarly. Property (P1) is equivalent to the solution set of $|\boldsymbol{Q}(\theta)|=0$ is $\Omega_{\beta}$. According to definitions of $\boldsymbol{Q}(b)$ in $(2)$ and $\boldsymbol{F}(b)$ in A.2), we obtain

$$
\boldsymbol{F}(b) \boldsymbol{Q}(b)=\left[\begin{array}{cc}
1 & \boldsymbol{b}_{[N-1]}^{T} \\
\mathbf{0} & \boldsymbol{E}_{N-1}\left(b^{N}\right)
\end{array}\right] .
$$

By using Lemma Appendix A.1 $|\boldsymbol{Q}(b)|=|\boldsymbol{F}(b) \boldsymbol{Q}(b)|=\left|\boldsymbol{E}_{N-1}\left(b^{N}\right)\right|=(1-$ 400 $\left.b^{N}\right)^{\left\lceil\frac{N-1}{2}\right\rceil}\left(1+b^{N}\right)^{\left\lfloor\frac{N-1}{2}\right\rfloor}$. Hence, $|\boldsymbol{Q}(b)|=0$ if and only if $b^{N}=e^{j N \pi \beta \sin (\theta)}= \pm 1$. Obviously, the solution set of $\theta$ is $\Omega_{\beta}$ for $|\theta| \in \Omega_{\theta}$. 
Property (P2) are proved in three steps: the first two show that $\operatorname{rank}\left(\boldsymbol{Q}_{[n+1]}(b)\right)$ $=n+1$ and $\operatorname{rank}(\boldsymbol{Q}(b))=n+1$ if $\theta \in \Omega_{\beta}$, and the last one derives $\operatorname{rank}\left(\boldsymbol{Q}_{[L]}(b)\right)$.

Step 1: When $L=n+1$, inserting A.1] into A.4, one can easily find that

to Step 2, if $\theta \in \Omega_{\beta}, \operatorname{rank}\left(\boldsymbol{Q}_{[L]}(\theta)\right) \leq \operatorname{rank}(\boldsymbol{Q}(\theta))=n+1$. Therefore, when $n+1 \leq L \leq N$ and $\theta \in \Omega_{\beta}$, we also have $\operatorname{rank}\left(\boldsymbol{Q}_{[L]}(\theta)\right)=n+1=\min (L, n+1)$.

\section{Appendix B. Proof of Lemma 4.1}

Property (P5): According to $111 \mathrm{~b}), \boldsymbol{H}\left(\boldsymbol{\theta}_{[2]}\right)$ is rank deficient only if both $\boldsymbol{Q}_{[L]}\left(\theta_{1}\right)$ and $\boldsymbol{Q}_{[L]}\left(\theta_{2}\right)$ are. This implies that $\theta_{m} \in \Omega_{\beta}, m=1,2$, according to Lemma 3.1. Moreover, following the definition of $\Omega_{\beta}, \sin \left(\theta_{2}\right)-\sin \left(\theta_{1}\right)=$ $\frac{k_{2}-k_{1}}{N \beta}=\frac{i}{N \beta}, i \in \mathbb{Z}$ and $|i| \leq 2 N \beta$. Comparing this with the definition of $\Omega_{\beta}^{2}$, we only need to prove that $\boldsymbol{H}\left(\boldsymbol{\theta}_{[2]}\right)$ is full column rank when $i=2 k+1$. Moreover, since $\boldsymbol{H}\left(\boldsymbol{\theta}_{[2]}\right)$ with $L<N$ is the first $L$ columns of the $\boldsymbol{H}\left(\boldsymbol{\theta}_{[2]}\right)$ with $L=N$. we only need to consider the $L=N$ case. Use notations in Appendix A and let

$$
\boldsymbol{F}^{(2)}=\operatorname{blkdg}\left[\boldsymbol{F}\left(b_{1}\right), \boldsymbol{F}\left(b_{2}\right)\right]
$$

Obviously, $\left|\boldsymbol{F}^{(2)}\right|=1$. When $L=N$, we can obtain

$$
\boldsymbol{F}^{(2)} \boldsymbol{H}\left(\boldsymbol{\theta}_{[2]}\right)=\left[\begin{array}{cccc}
1 & \mathbf{0}^{T} & 1 & \mathbf{0}^{T} \\
\boldsymbol{b}_{1,[N-1]} & \boldsymbol{E}_{N-1}^{T}\left(b_{1}^{N}\right) & \boldsymbol{b}_{2,[N-1]} & \boldsymbol{E}_{N-1}^{T}\left(b_{2}^{N}\right)
\end{array}\right]^{T} .
$$

Since $\theta_{1}, \theta_{2} \in \Omega_{\beta}$ and $i=k_{2}-k_{1}=2 k+1$, then $b_{1}^{N}=-b_{2}^{N}= \pm 1$. W.l.o.g., suppose $b_{1}^{N}=-b_{2}^{N}=-1$. Then $\boldsymbol{E}_{N-1}\left(b_{1}^{N}\right)=\boldsymbol{E}_{N-1}(-1)$ and $\boldsymbol{E}_{N-1}\left(b_{2}^{N}\right)=$ $\boldsymbol{E}_{N-1}(1)$. By using Lemma Appendix A.1, simply derivations will lead to

$$
\operatorname{rank}\left(\left[\left(\boldsymbol{E}_{N-1}(-1)\right)^{T},\left(\boldsymbol{E}_{N-1}(1)\right)^{T}\right]\right)=N-1 .
$$


Based on B.2 and B.3, $\operatorname{rank}\left(\boldsymbol{F}^{(2)} \boldsymbol{H}\left(\boldsymbol{\theta}_{[2]}\right)\right)=\operatorname{rank}\left(\boldsymbol{H}\left(\boldsymbol{\theta}_{[2]}\right)\right)=N$.

Property (P6): When $\boldsymbol{\theta}_{[2]} \in \Omega_{\beta}^{2}, b_{1}^{N}=b_{2}^{N}$. Inserting it into $\left.\mathrm{B} .2\right]$ and using linear algebra, we can obtain ( $\mathrm{P} 6$ ).

\section{Appendix C. Proof of Lemma 4.3}

We first introduce the following lemma.

Lemma Appendix C.1. Denote $b_{k}=e^{j \pi \beta \sin \left(\theta_{k}\right)}$,

$$
\begin{aligned}
\boldsymbol{F}\left(b_{k}\right) \boldsymbol{C A} & =\left[\boldsymbol{d}_{k}, \boldsymbol{D}_{k}^{T}\right]^{T} \in \mathbb{C}^{N \times M}, \\
\boldsymbol{D}^{(K)} & =\left[-\operatorname{blkdg}\left(\boldsymbol{D}_{1}, \boldsymbol{D}_{2}, \ldots, \boldsymbol{D}_{K-1}\right), \mathbf{1}_{K-1} \otimes \boldsymbol{D}_{K}\right],
\end{aligned}
$$

where $\boldsymbol{F}\left(b_{k}\right)$ is defined in A.2. Note that $\boldsymbol{D}^{(K)} \in \mathbb{C}^{(N-1)(K-1) \times K M}$, then $\operatorname{rank}\left(\boldsymbol{D}^{(K)}\right) \leq \min [(N-1)(K-1), M K,(M+2)(K-1)]$.

Proof. We only need to prove $\operatorname{rank}\left(\boldsymbol{D}^{(K)}\right) \leq(M+2)(K-1)$, which is achieved by finding a full rank $\boldsymbol{T}_{K} \in \mathbb{C}^{K M}$ such that $\operatorname{rank}\left(\boldsymbol{D}^{(K)} \boldsymbol{T}_{K}\right) \leq(M+2)(K-1)$.

First, we show the relations between columns of $\boldsymbol{D}_{k}$ and $\boldsymbol{D}_{l}$. According to the definition of $\boldsymbol{F}\left(b_{k}\right)$, then $\forall k, l \in[K], k \neq l$, and $\forall m \in[M]$, there is

$$
\left(a_{m}-b_{k}\right) \boldsymbol{F}\left(b_{l}\right)-\left(a_{m}-b_{l}\right) \boldsymbol{F}\left(b_{k}\right)=\left(b_{l}-b_{k}\right) \boldsymbol{F}\left(a_{m}\right),
$$

where $a_{m}=e^{j \pi \beta \sin \left(\tilde{\theta}_{m}\right)}$. Denote $\boldsymbol{c}_{f}=\left[c_{2}, \ldots, c_{N}\right]^{T}$ and $\boldsymbol{c}_{b}=\left[c_{N}, \ldots, c_{2}\right]^{T}$. Using A.4 and A.1, we have

$$
\left[\left(a_{m}-b_{k}\right) \boldsymbol{F}\left(b_{l}\right)-\left(a_{m}-b_{l}\right) \boldsymbol{F}\left(b_{k}\right)\right] \boldsymbol{C a}\left(\tilde{\theta}_{m}\right)=\left(b_{l}-b_{k}\right)\left[\boldsymbol{c}^{T} \boldsymbol{a}\left(\tilde{\theta}_{m}\right), \boldsymbol{c}_{f}^{T}-\boldsymbol{c}_{b}^{T} a_{m}^{N}\right]^{T} .
$$

Denoting $\boldsymbol{d}_{k, m}$ as the $m$-th column of $\boldsymbol{D}_{k}$ and substituting it into (C.4 , we have

$$
\left(a_{m}-b_{k}\right) \boldsymbol{d}_{l, m}-\left(a_{m}-b_{l}\right) \boldsymbol{d}_{k, m}=\left(b_{l}-b_{k}\right)\left[\boldsymbol{c}_{b}, \boldsymbol{c}_{f}\right]\left[-a_{m}^{N}, 1\right]^{T} .
$$

Second, we find $\boldsymbol{T}_{K}$ in scenarios where $\theta_{k} \neq \tilde{\theta}_{m}$. Since $b_{k}-a_{m} \neq 0$, for arbitrary $k \in[K-1]$ and $m \in[M]$, define

$$
\boldsymbol{T}_{K}=\left[\begin{array}{cc}
\boldsymbol{I}_{(K-1) M} & {\left[\boldsymbol{T}_{K 1}, \ldots, \boldsymbol{T}_{K(K-1)}\right]^{T}} \\
\mathbf{0} & \boldsymbol{I}_{M}
\end{array}\right],
$$


where $\boldsymbol{T}_{K k}=\operatorname{diag}\left(\frac{a_{1}-b_{K}}{a_{1}-b_{k}}, \ldots, \frac{a_{M}-b_{K}}{a_{M}-b_{k}}\right)$. Clearly, $\boldsymbol{T}_{K}$ is a full rank matrix and

$$
\boldsymbol{D}^{(K)} \boldsymbol{T}_{K}=\left[-\operatorname{blkdg}\left(\boldsymbol{D}_{1}, \boldsymbol{D}_{2}, \ldots, \boldsymbol{D}_{K-1}\right), \tilde{\boldsymbol{D}}_{K-1}\right],
$$

where $\tilde{\boldsymbol{D}}_{K-1}=\left[\left(\boldsymbol{D}_{K}-\boldsymbol{D}_{1} \boldsymbol{T}_{K 1}\right)^{T}, \ldots,\left(\boldsymbol{D}_{K}-\boldsymbol{D}_{K-1} \boldsymbol{T}_{K(K-1)}\right)^{T}\right]^{T}$ and

$$
\begin{aligned}
& \boldsymbol{D}_{K}-\boldsymbol{D}_{k} \boldsymbol{T}_{K k}=\left[\boldsymbol{c}_{b}, \boldsymbol{c}_{f}\right] \boldsymbol{G}_{M} \boldsymbol{D}_{K k}, k \in[K-1] \\
& \boldsymbol{G}_{M}=\left[\begin{array}{cccc}
-a_{1}^{N} & -a_{2}^{N} & \ldots & -a_{M}^{N} \\
1 & 1 & \ldots & 1
\end{array}\right], \\
& \boldsymbol{D}_{K k}=\left(b_{K}-b_{k}\right)\left(\operatorname{diag}\left(a_{1}-b_{k}, \ldots, a_{M}-b_{k}\right)\right)^{-1}
\end{aligned}
$$

according to C.5. Inserting (C.8) into $\tilde{\boldsymbol{D}}_{K-1}$ leads to

$$
\tilde{\boldsymbol{D}}_{K-1}=\left(\boldsymbol{I}_{K-1} \otimes\left(\left[\boldsymbol{c}_{b}, \boldsymbol{c}_{f}\right] \boldsymbol{G}_{M}\right)\right)\left[\boldsymbol{D}_{K 1}^{T}, \ldots, \boldsymbol{D}_{K(K-1)}^{T}\right]^{T} .
$$

Since rank of $\boldsymbol{I}_{K-1} \otimes\left(\left[\boldsymbol{c}_{b}, \boldsymbol{c}_{f}\right] \boldsymbol{G}_{M}\right)$ is $2(K-1)$ in general, $\operatorname{rank}\left(\tilde{\boldsymbol{D}}_{K-1}\right) \leq 2(K-1)$. According to (C.7), we have $\operatorname{rank}\left(\boldsymbol{D}^{(K)}\right) \leq(K-1)(M+2)$.

Finally, we find $\boldsymbol{T}_{K}$, when $\exists M_{1} \geq 1$ such that $\theta_{m}=\tilde{\theta}_{m}, \forall m \in\left[M_{1}\right]$. Let

$$
\boldsymbol{T}_{K k}=\operatorname{diag}\left(\mathbf{0}_{M_{1}}^{T}, \frac{a_{M_{1}+1}-b_{K}}{a_{M_{1}+1}-b_{k}}, \ldots, \frac{a_{M}-b_{K}}{a_{M}-b_{k}}\right) .
$$

Define $\boldsymbol{T}_{K}$ as in (C.6) and then switch its $((m-1) M+m)$-th and $((K-1) M+m)$ th columns $\forall m \in\left[M_{1}\right]$. Then simple derivations will show that $\operatorname{rank}\left(\boldsymbol{D}^{(K)}\right)=$ $\operatorname{rank}\left(\boldsymbol{D}^{(K)} \boldsymbol{T}_{K}\right) \leq(M+2)(K-1)$. The lemma is thus proved.

Define an elementary matrix $\boldsymbol{T}_{E}$ which substitutes the $(m+N)$-th row of a matrix by the difference between the $(m+N)$-th and the $m$-th rows, $\forall m=$ ${ }_{430} 2, \ldots, N$. Substituting B.2 and C.1 into $\boldsymbol{F}_{E}=\boldsymbol{T}_{E} \boldsymbol{F}^{(2)} \boldsymbol{H}_{E}\left(\boldsymbol{\theta}_{[2]}\right)$ and then making use of $\boldsymbol{E}_{N-1,[L-1]}\left(b_{2}^{N}\right)-\boldsymbol{E}_{N-1,[L-1]}\left(b_{1}^{N}\right)=\mathbf{0}, \forall \boldsymbol{\theta}_{[2]} \in \Delta_{\beta}^{2}$. One can obtain $\operatorname{rank}\left(\boldsymbol{H}_{E}\left(\boldsymbol{\theta}_{[2]}\right)\right)=\operatorname{rank}\left(\boldsymbol{F}_{E}\right) \leq N+1+\operatorname{rank}\left(\boldsymbol{D}^{(2)}\right)$. According to Lemma Appendix C.1 $\operatorname{rank}\left(\boldsymbol{D}^{(2)}\right) \leq M+2$. Hence, Lemma 4.3 is obtained.

\section{Appendix D. Proof of Lemma 5.1}

The first property is because that $P_{3 N}(\theta)$ equals the optimal value of problem

$$
\max _{\boldsymbol{v}_{[L]}} 1 /\left\|\boldsymbol{W}(\theta) \boldsymbol{v}_{[L]}\right\|^{2}, \text { s.t. }\left\|\boldsymbol{Q}_{[L]}(\theta) \boldsymbol{v}_{[L]}\right\|^{2}=1 .
$$


Denote a true DoA as $\tilde{\theta}$ and $\tilde{\theta} \notin \Omega_{\beta}$ (whose probability is 1 in practice), then $\tilde{\lambda}=\lambda_{1}\left(\boldsymbol{Q}_{[L]}^{H}(\tilde{\theta}) \boldsymbol{Q}_{[L]}(\tilde{\theta})\right)>0$ based on Lemma 3.1. Assume that a peak at $\tilde{\theta}$ is detected in spectrum $P_{3}(\theta)$ with a mutual coupling estimate $\tilde{\boldsymbol{v}}$ and $\|\tilde{\boldsymbol{v}}\|=1$. Then $\left\|\boldsymbol{Q}_{[L]}(\tilde{\theta}) \tilde{\boldsymbol{v}}\right\|^{2} \geq \tilde{\lambda}$ and $\left\|\boldsymbol{U}_{n}^{H} \boldsymbol{Q}_{[L]}(\tilde{\theta}) \tilde{\boldsymbol{v}}\right\|^{2} \approx 0$ if $N_{T}$ is large enough. Their ratio will cause a peak at $\tilde{\theta}$ in spectrum $P_{3 N}(\theta)$.

Finally, denote the optimal solution by $\tilde{\boldsymbol{v}}$ and assume $\tilde{\boldsymbol{v}} \neq \zeta \boldsymbol{c}_{[L]}$, where $\zeta \neq 0$ is a complex. Then $\frac{\left\|\boldsymbol{Q}_{[L]}(\tilde{\theta}) \tilde{\boldsymbol{v}}\right\|^{2}}{\|\boldsymbol{W}(\tilde{\theta}) \tilde{\boldsymbol{v}}\|^{2}}>\frac{\left\|\boldsymbol{Q}_{[L]}(\tilde{\theta}) \boldsymbol{c}_{[L]}\right\|^{2}}{\left\|\boldsymbol{W}(\tilde{\theta}) \boldsymbol{c}_{[L]}\right\|^{2}}$. Since $\tilde{\lambda}>0$, rescaling $\tilde{\boldsymbol{v}}$ (such that $\left.\left\|\boldsymbol{Q}_{[L]}(\tilde{\theta}) \tilde{\boldsymbol{v}}\right\|=\left\|\boldsymbol{Q}_{[L]}(\tilde{\theta}) \boldsymbol{c}_{[L]}\right\|\right)$ leads to $\|\boldsymbol{W}(\tilde{\theta}) \tilde{\boldsymbol{v}}\|^{2}<\left\|\boldsymbol{W}(\tilde{\theta}) \boldsymbol{c}_{[L]}\right\|^{2}$. When $N_{t}$ is large enough, $\left\|\boldsymbol{W}(\tilde{\theta}) \boldsymbol{c}_{[L]}\right\| \approx 0$ and hence $\|\boldsymbol{W}(\tilde{\theta}) \tilde{\boldsymbol{v}}\|^{2} \approx 0$, which means that the optimal solution, obtained by eigenvalue decomposition, is not unique.

[1] H. Krim, M. Viberg, Two decades of array signal processing research: the parametric approach, IEEE Signal Process. Mag. 13 (4) (1996) 67-94.

[2] C. A. Balanis, Antenna Theory: Analysis and Design, 4th Edition, Wiley, New York, NY, USA, 2016.

[3] I. Gupta, A. Ksienski, Effect of mutual coupling on the performance of adaptive arrays, IEEE Trans. Antennas Propag. 31 (5) (1983) 785-791.

[4] T. E. Tuncer, B. Friedlander, Classical and modern direction-of-arrival estimation, Academic Press, 2009.

[5] S. Qin, Y. D. Zhang, M. G. Amin, Generalized coprime array configurations for direction-of-arrival estimation, IEEE Trans. Signal Process. 63 (6) (2015) 1377-1390.

[6] J. Pierre, M. Kaveh, Experimental performance of calibration and direction-finding algorithms, in: Proc. IEEE ICASSP, 1991, pp. 1365-1368.

[7] B. C. Ng, C. M. S. See, Sensor-array calibration using a maximumlikelihood approach, IEEE Trans. Antennas Propag. 44 (6) (1996) 827-835.

[8] B. Porat, B. Friedlander, Accuracy requirements in off-line array calibration, IEEE Trans. Aerosp. Electron. Syst. 33 (2) (1997) 545-556. 
[9] T. Takahashi, N. Nakamoto, M. Ohtsuka, T. Aoki, Y. Konishi, I. Chiba, M. Yajima, On-board calibration methods for mechanical distortions of satellite phased array antennas, IEEE Trans. Antennas Propag. 60 (3) (2012) 1362-1372.

[10] Z. Ye, J. Dai, X. Xu, X. Wu, DoA estimation for uniform linear array with mutual coupling, IEEE Trans. Aerosp. Electron. Syst. 45 (1) (2009) 280-288.

[11] Z. Ye, C. Liu, On the resiliency of MUSIC direction finding against antenna sensor coupling, IEEE Trans. Antennas Propag. 56 (2) (2008) 371-380.

[12] B. Liao, Z. G. Zhang, S. C. Chan, DoA estimation and tracking of ulas with mutual coupling, IEEE Trans. Aerosp. Electron. Syst. 48 (1) (2012) 891-905.

[13] B. Friedlander, A. Weiss, Direction finding in the presence of mutual coupling, IEEE Trans. Antennas Propag. 39 (3) (1991) 273-284.

[14] F. Sellone, A. Serra, A novel online mutual coupling compensation algorithm for uniform and linear arrays, IEEE Trans. Signal Process. 55 (2) (2007) 560-573.

[15] Z. Liu, Y. Zhou, A unified framework and sparse bayesian perspective for direction-of-arrival estimation in the presence of array imperfections, IEEE Trans. Signal Process. 61 (15) (2013) 3786-3798.

[16] C. Qi, Y. Wang, Y. Zhang, H. Chen, DoA estimation and self-calibration algorithm for uniform circular array, Electron. Lett. 41 (20) (2005) 10921094 .

[17] M. Lin, L. Yang, Blind calibration and DoA estimation with uniform circular arrays in the presence of mutual coupling, IEEE Antennas Wirel. Propag. Lett. 5 (1) (2006) 315-318. 
[18] D. Gao, B. Wang, Y. Guo, Comments on Blind calibration and DoA estimation with uniform circular arrays in the presence of mutual coupling, IEEE Antennas Wirel. Propag. Lett. 5 (1) (2006) 566-568.

[19] J. Dai, X. Bao, N. Hu, C. Chang, W. Xu, A recursive RARE algorithm for DoA estimation with unknown mutual coupling, IEEE Antennas Wirel. Propag. Lett. 13 (2014) 1593-1596.

[20] Z. Liu, Z. Huang, F. Wang, Y. Zhou, DoA estimation with uniform linear arrays in the presence of mutual coupling via blind calibration, Signal Processing 89 (7) (2009) 1446-1456.

[21] M. Haardt, M. Pesavento, F. Roemer, M. N. E. Korso, Chapter 15 - subspace methods and exploitation of special array structures, in: A. M. Zoubir, M. Viberg, R. Chellappa, S. Theodoridis (Eds.), Academic Press Library in Signal Processing, Vol. 3, Elsevier, 2014, pp. $651-717$.

[22] S. Cai, A normalized spatial spectrum for DoA estimation with uniform linear arrays in the presence of unknown mutual coupling, in: Proc. IEEE ICASSP, 2016, pp. 3086-3090.

[23] B. Friedlander, Array self-calibration with large initial errors, in: Proc. IEEE ACSSC, 2014, pp. 1169-1173.

[24] G. H. Golub, C. F. V. Loan, Matrix Computations, 4th Edition, Johns Hopkins University Press, Baltimore, Maryland, USA, 2012.

[25] Y. Huang, D. Palomar, Rank-constrained separable semidefinite programming with applications to optimal beamforming, IEEE Trans. Signal Process. 58 (2) (2010) 664-678. 